\title{
Arctiini Leach, [1815] (Lepidoptera, Erebidae, Arctiinae) of the Brazilian Amazon. III - Subtribe Ctenuchina Kirby, 1837
}

\author{
José A. Teston ${ }^{1 *}$, Débora S. de Abreu ${ }^{1}$ \& Viviane G. Ferro ${ }^{2}$ \\ ${ }^{1}$ Universidade Federal do Oeste do Pará, Instituto de Ciências da Educação, Laboratório de Estudos de \\ Lepidópteros Neotropicais, Rua Vera Paz s/n, CEP 68040-255, Santarém, PA, Brasil \\ ${ }^{2}$ Universidade Federal de Goiás, Instituto de Ciências Biológicas, Departamento de Ecologia, Caixa Postal \\ 131, CEP 74001-970, Goiânia, GO, Brasil \\ *Corresponding author: José A. Teston, e-mail: jateston@gmail.com
}

TESTON, J. A., ABREU, D.S.; FERRO, V.G. Arctiini Leach, [1815] (Lepidoptera, Erebidae, Arctiinae) of the Brazilian Amazon. III - Subtribe Ctenuchina Kirby, 1837. Biota Neotropica. 19(2): e20180673. http://dx.doi.org/10.1590/1676-0611-BN-2018-0673

\begin{abstract}
The Ctenuchina moths comprise 874 New World species. Here we provide a list of Ctenuchina species from the Brazilian Amazon. The list was produced from specimens deposited in the most important Brazilian collections and from literature data. Nearly 31\% (273) of the New World Ctenuchina species were recorded, including 28 new occurrences for the Brazilian Amazon. Santarém and Belém were the municipalities with the highest number of species records, with 139 and 88, respectively. The high number of Ctenuchina records in the Amazon is underestimated because this subtribe has never been sampled in the vast majority of the biome. This is a worrying scenario because the Amazon has the world highest absolute rate of forest reduction. To suggest efficient conservation policies for the Arctiinae fauna of Amazon, it is urgent to intensify the sampling effort in this biome. Keywords: Amazon, Tiger moths, Inventory, Noctuoidea, Wasp Moths.
\end{abstract}

\section{Arctiini Leach, [1815] (Lepidoptera, Erebidae, Arctiinae) da Amazônia Brasileira. III - Subtribo Ctenuchina Kirby, 1837}

\begin{abstract}
Resumo: As mariposas Ctenuchina compreendem 874 espécies no Novo Mundo. Neste trabalho, nós apresentamos uma lista das espécies de Ctenuchina que ocorrem na Amazônia brasileira. A lista foi produzida através de observação de espécimes depositados nas mais importantes coleções brasileiras e também através de dados da literatura. Aproximadamente 31\% (273) das espécies de Ctenuchina que ocorrem no Novo Mundo foram registradas, incluindo 28 novas ocorrências para a Amazônia brasileira. Santarém e Belém foram os municípios com maior número de espécies registradas, com 139 e 88, respectivamente. Embora o número de registros de Ctenuchina seja considerado alto na Amazônia, este valor é subestimado visto que a fauna nunca foi amostrada na imensa maioria do bioma. Este cenário é preocupante, pois a Amazônia possui a maior taxa absoluta de redução florestal do mundo. A fim de podermos sugerir políticas de conservação eficientes para a fauna de Arctiinae da Amazônia, é urgente que se intensifique o esforço de coleta neste bioma.

Palavras-chave: Amazônia, mariposas-tigre, Inventário, Noctuoidea, mariposas-vespa.
\end{abstract}

\section{Introduction}

Arctiinae is distributed worldwide (Heppner 1991). With approximately 11,000 species, the Arctiinae moths are divided in four tribes (Zahiri et al. 2012), but only Arctiini and Lithosiini occurr in the Neotropics. Arctiini is classified in seven subtribes: Arctiina, Callimorphina, Ctenuchina, Euchromiina, Phaegopterina, Pericopina, and Spilosomina (Weller et al. 2009, Vincent \& Laguerre 2014).

The Ctenuchina moths are found in the "New World" and comprise 874 species (Weller et al. 2009). Several species have nocturnal activity, but some species are exclusively diurnal (Hagmann 1938). Many species of Ctenuchina form mimicry rings with several wasp species (Simmons, 2009). They have a pair of tympanic organs located in the metathorax, above the spiracles, covered by an operculum. The hind wings are small or reduced, with absence of $\mathrm{S}_{\mathrm{c}}+\mathrm{R}_{1}$ veins and separated $\mathrm{C}_{\mathrm{u}} \mathrm{A}$ branches (Kitching \& Rawlins 1999, Jacobson \& Weller 2002). The larvae feed on Apocynaceae, Euphorbiaceae, Ebenaceaa, Moraceae, Fabaceae, Ulmaceae, Poaceae, Loganiaceae, Urticaceae, Malpighiaceae, Vochysiaceae, Malvaceae, Ochnaceae and Cyperaceae (Janzen \& Hallwachs 2005). The adults of several species are pharmacophagous and feed on pyrrolizidine alkaloids (Conner \& Jordan 2009). 
We here report a list of Ctenuchina moths occurring in the Brazilian Amazon based on the specimens deposited in the most important Brazilian collections and also from literature data. This study is a continuation of Teston \& Ferro (2016a, b) and aims to increase the knowledge on Arctiinae in the Amazon region.

\section{Materials and methods}

We intensively searched the literature and examined specimens from entomological collections of the Instituto Nacional de Pesquisas na Amazônia (INPA; Manaus), Museu Paraense Emilio Goeldi (MPEG; Belém), Coleção Becker (VOB; Camacan), Coleção Entomológica Padre Jesus Santiago Moure of the Universidade Federal do Paraná (DZUP; Curitiba), Fundação Instituto Oswaldo Cruz (FIOC; Rio de Janeiro), Museu de Zoologia of the Universidade de São Paulo (MZUSP; São Paulo), Museu Nacional of the Universidade Federal do Rio de Janeiro (MNRJ; Rio de Janeiro), and Laboratório de Estudos de Lepidópteros Neotropicais (LELN) of the Universidade Federal do Oeste do Pará (UFOPA; Santarém). To identify the species, we used literature (Hampson 1898, 1914, Seitz 1919-1925) and specimens deposited in the visited collections. The systematic organization to generic level follows Hampson (1898) and Weller et al. (2000), with updates of the generic names according Watson et al. (1995) and corrections of Pinheiro \& Duarte (2013), Pinheiro \& Gaal-Haszler (2015) and Pinheiro (2016).

The geographical coordinates of the localities in the Brazilian Amazon with Arctiinae records were obtained from the Geo Loc tool of "Species Link date \& tools" (http://splink.cria.org.br/geoloc) and Google Earth (https://earth.google.com/web/). The list is organized alphabetically. Species and records without precise location data, and those from locations that belong to more than one biome (e.g., Cerrado and Amazon) were not included in the list.

\section{Results}

Our research generated a list of 273 Ctenuchina species (Table 1), including 28 new occurrences for the Brazilian Amazon (indicated by "NEW"). Twenty-three species appear as new records for the municipalities and their respective States (indicated by "AMZ"). In total, 75 (nearly 10\%) Amazonian municipalities had Ctenuchina species records (Table 2 and Figure 1). Santarém (PA), Belém (PA), and Cacaulândia $(\mathrm{RO})$ were the municipalities with the highest number of species, with 139,88 , and 60 , respectively.

We recorded 61 genera, of which 20 were monospecific. The genera Eucereon Hübner, 1819 had the highest number of species (37), followed by Delphyre Walker, 1854 (20), and Heliura Butler, 1876 (17). The species with the highest number of locality records were Eucereon varia (Walker, 1854) (with 23), followed by Telioneura glaucopis R. Felder, 1869 (22), Correbidia calopteridia (Butler, 1878) (21) and Epidesma ursula (Cramer, 1782) (20). Eighty-nine species (32.6\%) occurred in only one locality (Table 1).

\section{Discussion}

The number of Ctenuchina species recorded for the Brazilian Amazon reported on this study is high. It corresponds to $31.2 \%$ of "New World" Ctenuchina fauna (Weller et al. 2009) and is more than two times higher than what has been reported for Mexico (Hernández-Baz et al. 2013) and the Brazilian Cerrado (Ferro et al. 2010). The fact that Santarém and Belém are the Amazon municipalities with the highest number of Ctenuchina records is probably related to the proximity and access to the collection sites. Belém is the capital of the state of Pará and has an important museum (Museu Paraense Emílio Goeldi, founded in 1866) and other research institutions, as well as easy access to sampling sites. Santarém has been widely sampled by H. Zerny and G. Hagmann in the 1930s (Zerny 1931, Hagmann 1938). Hagmann resided in Santarém and Zerny spent a season collecting in forests and other natural areas nearby Santarém. Belém is also the second municipality with the highest number of Phaegopterina and Pericopina records (Teston \& Ferro 2016a, b).

The Ctenuchina richness of Santarém and Belém is the largest recorded in Brazil. For example, in Salesópolis it has been recorded 42 Ctenuchina species (Ferro \& Diniz 2007) and in Joinville 57 species (Ferro et al. 2012). These two last sites are located in the Atlantic Forest biome and were intensively sampled in the recent past. In the Cerrado sites, a much lower number of Ctenuchina species has been recorded, ranging from 3 (Scherrer et al. 2013) to 26 (Moreno \& Ferro 2016). Moreover, the number of Ctenuchina species in a single Amazon site (Santarém, 139) is higher than that recorded for the whole Cerrado (133 species, Ferro et al. 2010). It may indicate that this taxon is more diverse in rain-forest than in xeric environments.

Until now Teston and Ferro (2016b) have reported 819 Arctiinae species in the Brazilian Amazon. Due to the new records reported in this study (28) there has been an increase in richness for the biome, and now a total of 847 tiger moth species are recorded for the Amazon. Similarly to the the subtribes Phaegopterina (Teston \& Ferro 2016a) and Pericopina (Teston \& Ferro 2016b), the number of Amazonian species of Ctenuchina is underestimated because it has never been sampled in the vast majority of the biome (less than $10 \%$ of the municipalities has been sampled until now). In addition, the sampling points are poorly distributed and usually restricted to more populated cities and along major rivers. This is a worrying scenario because the Amazon has the world highest absolute rate of forest reduction (Silva et al. 2005). This biome has suffered from logging, fires, fragmentation, mining, wildlife extinction, invasion of alien species, and wildlife trafficking (Fearnside 2003). To suggest efficient conservation policies for the Arctiinae moths in the Amazon, it is urgent to intensify the collection effort in this biome. 
Table 1. Ctenuchina (Erebidae, Arctiinae, Arctiini) species of the Brazilian Amazon. The column with the records shows the Brazilian state in abbreviated form followed by municipality. The name of the locality is enclosed in braces and the author of the first record is in parentheses. * New record. States abbreviations: $\mathrm{AC}=\mathrm{Acre}, \mathrm{AM}=$ Amazonas, $\mathrm{AP}=$ Amapá, $\mathrm{MA}=$ Maranhão, $\mathrm{MT}=$ Mato Grosso, $\mathrm{PA}=$ Pará, $\mathrm{RO}=$ Rondônia and $\mathrm{RR}=$ Roraima.

\begin{tabular}{ll}
\hline & Species \\
\hline 1. & Abrochia aequalis (Walker, [1865]) \\
& \\
2. & Abrochia atridorsata (Hampson, 1909) \\
3. & Abrochia aurantivena (Hampson, 1918) \\
4. & Abrochia consobrina (Walker, 1856) \\
5. & Abrochia discoplaga (Schaus, 1905)
\end{tabular}

6. Abrochia eumenoides (Gaede, 1926)

7. Abrochia fulvisphex (Druce, 1898)

8. Abrochia mellina (Herrich-Schäffer, 1855)

9. $\quad$ Abrochia munda (Walker, 1856)

10. Abrochia postica (Walker, 1854)

11. Abrochia singularis (Walker, 1854)

12. Abrochia zethus (Hübner, 1827)

13. Aclytia albistriga Schaus, $1910^{\mathrm{AmZ}}$

14. Aclytia apicalis (Walker, 1854)

15. Aclytia gynamorpha Hampson, 1898

16. Aclytia heber (Cramer, [1780])

\section{Record}

AM, Tefé \{Ega\} (Walker [1865]); AP, Serra do Navio*; MA, Açailândia*; PA, Altamira \{Serra do Pardo National Park\} (Teston \& Correa 2015), Santarém \{Taperinha\} (Zerny 1931), São Félix do Xingu \{Serra do Pardo National Park\} (Teston \& Correa 2015)

AM, São Gabriel [da Cachoeira] \{Taracuá\} (Bryk 1953)

PA, [Belém] (Zerny 1931)

[AM], Valley of Amazonas [river] (Zerny 1931); PA, [Belém] (Walker 1856), Tapajós [river] (Hampson 1898)

MA, Açailândia*; MT, Sinop*; PA, Altamira \{Serra do Pardo National Park\} (Teston \& Correa 2015), Santarém \{Taperinha\} (Zerny 1931), São Félix do Xingu \{Serra do Pardo National Park\} (Teston \& Correa 2015); RO, Cacaulândia*

AM, [Boa Vista do Ramos] \{Massauari\} (Gaede 1926)

AM, Manaus (Bryk, 1953); MT, Sinop*; PA, Altamira \{Serra do Pardo National Park\} (Teston \& Correa 2015), Santarém \{Taperinha\} (Zerny 1931), São Félix do Xingu \{Serra do Pardo National Park\} (Teston \& Correa 2015); RO, Cacaulândia*

PA, Cametá (Hampson 1898)

PA, [Belém] (Walker 1854a), Santarém (Hampson 1898)

PA, [Belém] (Walker 1854a), Santarém (Hampson 1898)

PA, [Belém] (Hampson 1898)

PA, [Belém] (Walker 1854a), Santarém (Hampson 1898), Tapajós [river] (Walker 1854a)

PA, Belém*, Capitão Poço*

PA, [Belém] (Walker 1854a)

AM, [Jutaí] \{Jutaí river\} (Hampson 1898), Juruá river (Hampson 1898), Santa Isabel do Rio Negro*; PA, Belém \{Miramar\}*, Belterra \{National Forest of Tapajós\} (Freitas 2014), Breves*, Marabá* Santarém (Hampson 1898), Santarém \{Taperinha\} (Zerny 1931)

AM, [Carauari] \{Juruá river, [Lake of] Pupunha\} (Butler 1878), [Jutaí] \{Jutaí river, Boa Vista\} (Butler 1878), [São Gabriel da Cachoeira] \{Taracuá\} (Bryk 1953); MA, Açailândia*; MT, Sinop*; [PA], lower Amazonas [river] (Druce 1884), PA, Altamira \{Serra do Pardo National Park\} (Teston \& Correa 2015), Belterra \{National Forest of Tapajós\} (Freitas 2014), Marabá*, Santarém (Butler 1876), São Félix do Xingu \{Serra do Pardo National Park\} (Teston \& Correa 2015)

AM, Santa Isabel do Rio Negro*; PA (Hagmann 1938), Belém \{Miramar\}*, Santarém*; RO, Porto Velho*

PA, Santarém*; RO, Ariquemes*, Cacaulândia*

AM, Manicoré*; MA, Açailândia*; PA, Altamira \{Serra do Pardo National Park\} (Teston \& Correa 2015), Belterra \{National Forest of Tapajós\} (Freitas 2014), Santarém (Hampson 1898), São Félix do Xingu \{Serra do Pardo National Park\} (Teston \& Correa 2015); RO, Cacaulândia*, Porto Velho*

AM, Manicoré \{Madeira river\} (Zerny 1931), São Gabriel da Cachoeira*; PA, Santarém (Hampson 1914)

MT, Sinop*; RO, Cacaulândia*

RO, Vila Rondônia*

AM, Borba*; AP, Mazagão*; PA, Benevides*, Curralinho*, Parauapebas \{Serra Norte, Carajás \}*, Santarém*; RO, Porto Velho*, RR, Alto Alegre*

AM, Manaus* 
Teston, J.A. et al.

Continuation Table 1.

\begin{tabular}{ll}
\hline & Species \\
\hline 25. & Agyrta auxo Hübner, 1817 \\
26. & Agyrta bifasciata Rothschild, 1912 \\
27. & Agyrta chena Druce, 1893 \\
28. & Agyrta dux (Walker, 1854) \\
& \\
29. & Agyrta klagesi Rothschild, 1912 \\
30. & Agyrta micilia (Cramer, 1780) \\
31. & Agyrta pandemia Druce, 1893 \\
32. & Agyrta porphyria (Cramer, 1782)
\end{tabular}

33. Agyrtidia olivensis Machado Filho e Rêgo Barros, 1970

34. Agyrtidia uranophila (Walker, 1866)

35. Agyrtiola niepelti Gaede 1926

36. Anaphlebia caudatula R. Felder, 1869

37. Androcharta diversipennis (Walker, 1854)

38. Androcharta hoffmannsi (Rothschild, 1912)

39. Androcharta meones (Cramer, 1780)

40. Androcharta stretchii Butler, 1876

41. Antichloris affinis (Rothschild, 1912)

42. Antichoris caca Hübner, 1818

43. Antichloris chloroplegia (Druce, 1905) NEW $^{\mathrm{NEW}}$

44. Antichloris eriphia (Fabricius, 1776)

45. Antichloris scudderii Butler, 1876

46. Argyroeides flavicornis Rothschild, 1911

\section{Record}

AM, São Paulo de Olivença*; AP, Serra do Navio*; PA, [Belém] (Zerny 1931), Belém \{Miramar\}*, Ourém*, Santarém*

PA, Santarém \{Mojú\} (Zerny 1931)

AM, Manaus (Bryk 1953), [São Gabriel da Cachoeira] \{Taracuá\} (Bryk 1953); PA, [Belém] (Druce 1893)

AM, [Eirunepé] \{Juruá river, São Felipe\} (Zerny 1931), Manicoré*, Maués*; AP, Serra do Navio*; PA, Anajás*, Belém*, Parauapebas \{Serra Norte, Carajás\}*, Óbidos*, Santarém \{Taperinha\} (Zerny 1931)

PA, Santarém*

AM, Manicoré*, Santa Isabel do Rio Negro*; PA, [Belém] (Hampson 1898); RO, Cacaulândia*, Candeias do Jamari*

PA, [Belém] (Druce 1893)

AM, [Eirunepé] \{Juruá river, São Felipe\} (Zerny 1931), Manaus (Zerny 1931), Manicoré*, [Maués] \{Rio Parauary\}*, São Paulo de Olivença*, Tefé*; AP, Santana*, Serra do Navio*; PA, [Belém] (Hampson 1898), Itaituba*, Santarém \{Taperinha\} (Zerny 1931); RO, Vila Rondônia*

AM, São Paulo de Olivença (Machado Filho \& Rêgo Barros 1970)

[AC], Alto Juruá (Machado Filho \& Rêgo Barros 1970); AM, [Autazes] \{Autaz river, Campinhas\} (Bryk 1953), Benjamin Constant*, Borba (Machado Filho \& Rêgo Barros 1970), [Eirunepé] \{Rio Juruá, São Felipe\} (Machado Filho \& Rêgo Barros 1970), [São Gabriel da Cachoeira] \{Rio Negro, Jauaretê\} (Machado Filho \& Rêgo Barros 1970), São Paulo [de Olivença] (Walker 1866); PA, Itaituba*, Santarém (Machado Filho \& Rêgo Barros 1970); RO, Cacaulândia*, Jarú*

AM, São Paulo de Olivença (Gaede 1926)

AM, Amazonas [river] (R. Felder 1869)

AM, Benjamin Constant*, [Carauari] \{Juruá river, [Lake of] Pupunha\} (Butler 1878), [Eirunepé] \{Juruá river, São Felipe\} (Zerny 1931), [Jutaí] \{Barreiras de Jutaí\} (Butler 1878), Manaus*, São Paulo [de Olivença] (Butler 1876), Tabatinga (Butler 1876), Tefé \{Ega\} (Butler 1876); AP, Serra do Navio*; PA, Itaituba*, Santarém \{Mojú and Taperinha\} (Zerny 1931), Tapajós [river] (Walker 1854a)

AM, Benjamin Constant*, Humaitá (Hampson 1914), São Paulo de Olivença*; RO, Cacaulândia*

AM, Negro river (Zerny 1931), São Paulo de Olivença (Santos 1939); PA, Altamira \{Monte Santo\} (Delfina \& Teston 2013), Santarém \{Mojú and Taperinha\} (Zerny 1931)

AM, Benjamin Constant*, Maués*, São Paulo [de Olivença] (Butler 1876); AP, Serra do Navio*; PA, Itaituba*, Santarém*, Tucuruí*

AM, Tefé (Rothschild 1912); PA (Hagmann 1938)

AM, São Paulo de Olivença*, Tefé (Field 1975); AP, Serra do Navio*; PA, [Belém] (Hampson 1898), Soure*

AM, São Paulo de Olivença*; AP, Serra do Navio*

AC, Feijó*; AM, Benjamin Constant*, Manaus*, Manicoré*, São Paulo de Olivença*, Tefé*; PA, [Belém] (Butler 1876), Altamira \{Monte Santo\} (Delfina $\&$ Teston 2013), Breves*, Santarém \{Taperinha\} (Zerny 1931), Tapajós [river] (Walker 1854a); RO, Ariquemes*, Cacaulândia*, Porto Velho*

AM, Itacoatiara*, Manaus (Zerny 1931), Manicoré*, São Paulo de Olivença*; PA, Oriximiná \{Rio Cuminá\}* , Santarém (Butler 1876), Santarém \{Taperinha\} (Zerny 1931)

AM, Tefé (Rothschild 1911) 
Continuation Table 1.

\begin{tabular}{ll}
\hline \multicolumn{2}{|c}{ Species } \\
\hline 47. & Argyroeides ophion (Walker, 1854) \\
48. & Argyroeides strigula Druce, $1896^{\mathrm{NEW}}$ \\
49. & Atyphopsis modesta Butler, 1878 \\
& \\
50. & Cacostatia buchwaldi (Rothschild, 1912) \\
51. & Cacostatia discalis (Walker, 1856) \\
52. & Cacostatia flaviventralis Dognin, 1909 \\
53. & Cacostatia germana (Rothschild, 1912) \\
54. & Ceramidia fumipennis (Walker, 1854)
\end{tabular}

55. Ceramidia phemonoides (Möschler, 1854)

56. Ceramidiodes obscurus (Butler, 1877)

57. Cercopimorpha dolens Schaus, 1905

58. Cercopimorpha homopteridia Butler, 1876

59. Cercopimorpha postflavida (Rothschild, 1912) NEW $^{\text {New }}$

60. Clystea andromacha (Fabricius, 1775)

61. Clystea aner (Hampson, 1905)

62. Clystea carnicauda (Butler, 1876)

63. Clystea daltha (Druce, 1895)

64. Clystea eburneifera (R. Felder, 1869)

65. Clystea gracilis (Möschler, 1878) AMZ $^{\mathrm{A}}$

66. Clystea leucaspis (Cramer, 1775)

67. Clystea stipata (Walker, 1854)

68. Corematura chrysogastra (Perty, 1834)

69. Correbia elongata Rothschild, 1912 AMZ

70. Correbia felderi Rothschild, 1912

71. Correbia lycoides (Walker, 1854)

72. Correbia obscura Schaus, $1905^{\mathrm{NEW}}$

73. Correbia tristitia Kaye, 1911

\section{Record}

AM, Manicoré*

MA, Santa Luzia*

AM, [Jutaí] \{Rio Jutaí, Boaventura\} (Butler 1878), Manicoré \{Rio Madeira\} (Zerny 1931); AP, Serra do Navio*; MT, Sinop*; PA, Belém*, Santarém (Butler 1878), Santarém \{Taperinha\} (Zerny 1931); RO, Porto Velho*

RO, [Porto Velho] \{Madeira river, Aliança\} (Rothschild 1912)

[AM], Valley on the Amazon [river] (Walker 1856)

PA, Almeirim \{Jari\} (Hawes et al. 2009)

AM, Manaus*; PA, Belém*

AM, Fonte Boa (Field 1975), Humaitá (Field 1975), Manaus (Field 1975), Manicoré \{Madeira river\} (Field 1975), [Novo Airão] \{Igarapé Preto\} (Field 1975), Solimões [river] (Bryk 1953), São Paulo de Olivença (Field 1975) Tefé $\{$ Ega $\}$ (Walker 1854a), Tonantins (Field 1975); PA, Tapajós river (Field 1975); RO, [Porto Velho] \{Rio Madeira, Aliança\} (Field 1975)

AM, Manaus (Zerny 1931); PA, Tapajós [river] (Butler 1876)

AM, Benjamin Constant*, Manaus*, Manicoré (Hampson 1898), [Tapauá] \{Rio Purus, [Lake] Sobral\} (Butler 1877), Tefé (Rothschild 1912)

MT, Sinop*; PA, Santarém \{Taperinha\} (Zerny 1931)

AM, Manicoré*; MT, Sinop*; PA, [Belém] (Walker 1854a), Santarém \{Taperinha\} (Zerny 1931)

PA, Santarém*; RO, Cacaulândia*

PA, [Belém] (Walker 1854a), Altamira \{Monte Santo\} (Delfina \& Teston 2013); $\mathrm{RR}$, Alto Alegre*

PA (Hagmann 1938)

PA, [Belém] (Hampson 1898), Altamira $\left\{51^{\circ} \mathrm{BIS}\right\}$ (Teston et al. 2012)

AM, Benjamin Constant*, [Eirunepé] \{Rio Juruá, Matto Pyri\} (Zerny 1931), Manaus*, Manicoré, São Paulo de Olivença*; PA, [Belém] (Druce 1895), Parauapebas $\{\text { Serra Norte, Carajás }\}^{*}$, Santarém*; RO, Cacaulândia*

AM, Amazonas [river] (R. Felder 1869), Tefé*

PA, Benevides*, Parauapebas \{Serra Norte, Carajás $\} *$

PA (Hagmann 1938)

AM, Santa Isabel do Rio Negro*; MA, Santa Luzia*; PA, [Belém] (Walker 1854a), [Igarapé-Miri] \{Estuary of Tocantins [river]\} (Zerny 1931), Parauapebas \{Serra Norte, Carajás\} *

AM, Benjamin Constant*, Borba*, Fonte Boa*, Rio Madeira (Butler 1878), [Santa Isabel do Rio Negro] \{Rio Preto ${ }^{*}$, São Paulo [de Olivença] (Butler 1876), São Paulo de Olivença \{Rio Solimões, S. Jerônimo\} (Travassos Filho 1952) and \{Rio Preto\} (Travassos Filho 1938), Tefé*

AM, Manaus*; MA, Açailândia*; PA, Novo Progresso \{Cachimbo\}*, Santarém* [AM], Amazonas (Rothschild 1912); PA, Santarém \{Taperinha\} (Zerny 1931)

AM, Barcelos \{Moura\}*, Benjamin Constant*, [Eirunepé] \{Rio Juruá, São Felipe (Zerny 1931), São Gabriel da Cachoeira \{Querari\}*; AP, Serra do Navio*;MT, Sinop*; PA, Altamira \{Monte Santo\} (Teston \& Delfina 2010), [Belém] (Walker 1854a), Belterra \{National Forest of Tapajós\} (Freitas 2014), Capitão Poço*, Igarapé-Açu*, Marabá*, Santarém \{Taperinha\} (Zerny 1931), São Félix do Xingu \{Serra do Pardo National Park\} (Teston \& Correa 2015); RO, Cacaulândia*, Porto Velho*; RR, Alto Alegre*

RO, Porto Velho*

AM, Codajás (Rothschild 1912), Fonte Boa (Rothschild 1912), PA, Santarém \{Taperinha\} (Zerny 1931) 
Teston, J.A. et al.

Continuation Table 1.

\begin{tabular}{ll}
\hline & Species \\
\hline 74. & Correbidia assimilis (Rothschild, 1912) \\
75. & Correbidia calopteridia (Butler, 1878) \\
& \\
& \\
& \\
76. & Correbidia germana (Rothschild, 1912) \\
77. & Correbidia notata (Butler, 1878) \\
78. & Correbidia simonsi (Rothschild, 1912) \\
79. & Correbidia steinbachi (Rothschild, 1912) \\
80. & Ctenucha circe (Cramer, 1780) \\
81. & Ctenucha mortia Schaus, 1901 \\
82. & Ctenucha tapajoza Dognin, 1923 \\
83. & Cyanopepla glaucopoides (Walker, 1854) \\
84. & Cyanopepla hurama (Butler, 1876) \\
85. & Delphyre aclytioides (Hampson, 1901) \\
87. & Delphyre albiventus (Druce, 1898) \\
& \\
& \\
& \\
& \\
& \\
8iscalis (Druce, 1905)
\end{tabular}

Record

AM, [Atalaia do Norte] \{Santo Antonio do Javari\} (Rothschild 1912), PA, Belterra \{National Forest of Tapajós\} (Freitas 2014), Santarém (Valente et al.2018)

AM, Barcelos $\{$ Moura $\} *$, Benjamin Constant*, Fonte Boa*, [Manaus] \{Rio Urubu $\}^{*}$, Santa Isabel do Rio Negro*, São Gabriel da Cachoeira \{Querari\} *, São Paulo de Olivença*,Tefé (Zerny 1931); AP, Serra do Navio*; MA, Açailândia*; MT, Aripuanã*, Sinop*; PA, Altamira \{Serra do Pardo National Park\} (Teston \& Correa 2015), [Belém] (Butler 1878), Belterra \{National Forest of Tapajós\} (Freitas 2014), Marabá*, Santarém \{Taperinha\} (Zerny 1931), São Félix do Xingu \{Serra do Pardo National Park\} (Teston \& Correa 2015); RO, Cacaulândia*, Porto Velho*

PA, Santarém \{Taperinha\} (Zerny 1931)

AM, Barcelos $\{\text { Moura }\}^{*}$, [Jutaí] \{Rio Jutaí\} (Butler 1878); PA, Altamira $\left\{51^{\circ} \mathrm{BIS}\right\}$ (Teston et al. 2012), Marabá*, Santarém \{Taperinha\} (Zerny 1931)

AM, Fonte Boa (Hampson 1914)

AM, Fonte Boa (Rothschild 1912)

PA, [Belém] (Walker 1854b)

RO, Candeias do Jamari*

PA, Tapajós river (Dognin 1923)

AM, [Eirunepé] \{Juruá river, São Felipe\} (Zerny 1931), Manaus*, Maués*; PA, [Belém] (Walker 1854b), Santarém*

PA, Itaituba (Staudinger 1894)

PA, Belterra \{National Forest of Tapajós\} (Freitas 2014)

AM (Zerny 1912); PA, Paragominas*

MT, Sinop*; PA, Altamira $\left\{51^{\circ}\right.$ BIS $\}$ (Teston et al. 2012), Santarém \{Taperinha\} (Zerny 1931), São Félix do Xingu \{Serra do Pardo National Park\} (Teston \& Correa 2015); RO, Cacaulândia*

88. Delphyre distincta (Rothschild, 1912)

89. Delphyre dizona (Druce, 1898)

RO, [Porto Velho] \{Madeira river, Aliança\} (Hampson 1914)

AM, Manicoré*; MA, Açailândia*; PA, Altamira \{Serra do Pardo National Park\} (Teston \& Correa 2015), Belém*, Belterra \{National Forest of Tapajós\} (Freitas 2014), Marabá*, Santarém \{Taperinha\} (Zerny 1931), São Félix do Xingu \{Serra do Pardo National Park\} (Teston \& Correa 2015); RO, Cacaulândia*, Porto Velho*

90. Delphyre fenestrata Bryk, 1953

91. Delphyre flaviceps (Druce, 1905)

AM, [São Gabriel da Cachoeira] \{Taracuá\} (Bryk 1953)

AM, Manicoré (Zerny 1931); PA, Belém*, Belterra \{National Forest of Tapajós\} (Freitas 2014), Marabá*, Santarém \{Taperinha\} (Zerny 1931), São Félix do Xingu \{Serra do Pardo National Park\} (Teston \& Correa 2015); RO, Cacaulândia*, [Porto Velho] \{Madeira river, Aliança and Calama\} (Rothschild 1912)

92. Delphyre flaviventralis Hampson, 1901

93. Delphyre hampsoni (Rothschild, 1912)

94. Delphyre hebes Walker, $1854^{\mathrm{Amz}}$

95. Delphyre macella (Dognin, 1911) NEW $^{\mathrm{NEW}}$

96. Delphyre maculosa (Hampson, 1898)

97. Delphyre meridionalis (Rothschild, 1912)

98. Delphyre minuta (Möeschler, 1877)

99. Delphyre parcipuncta Hampson, 1914

PA, Santarém \{Taperinha\} (Zerny 1931)

MA, Açailândia*; PA, São Félix do Xingu \{Serra do Pardo National Park\} (Teston \& Correa 2015); RO, [Porto Velho] \{Madeira river, Aliança\} (Rothschild 1912)

RO, Cacaulândia*

PA, Marabá*

AP, Oiapóque*; PA, [Belém] (Hampson 1898)

RO, [Porto Velho] \{Madeira river, Calama\} (Rothschild 1912)

PA, Santarém \{Taperinha\} (Zerny 1931)

PA, Altamira $\left\{51^{\circ} \mathrm{BIS}\right\}$ (Teston et al. 2012), [Breves] \{Furos de Breves $\}$ (Zerny 1931); RO, [Porto Velho] \{Madeira river, Aliança\} (Rothschild 1912) 
Continuation Table 1.

\begin{tabular}{ll}
\hline \multicolumn{2}{c}{ Species } \\
\hline 100. & Delphyre pieroides (Rothschild, 19 \\
101. & Delphyre pumila (Rothschild, 1912 \\
102. & Delphyre pusilla (Butler, 1878) \\
& \\
103. & Delphyre roseiceps Dognin, 1909 \\
104. & Delphyre varians Hampson, 1901 \\
105. Dinia aeagrus (Cramer, 1779)
\end{tabular}

106. Dinia mena (Hübner, [1827]) AMZ $^{\mathrm{A}}$

107. Diptilon aurantiipes Rothschild, 1911

108. Ecdemus carmania (Druce, 1883)

109. Ecdemus fuliginosa (Rothschild, 1912) NEW $^{\mathrm{N}}$

110. Ecdemus hypoleucus Herrich-Schäffer, [1855]

111. Ecdemus imbecillus (Zerny, 1931)

112. Ecdemus pereirai Travassos, $1940^{\mathrm{AMZ}}$

113. Ecdemus rubrothorax (Rothschild, 1911)

114. Epanycles imperialis (Walker, 1854)

115. Epidesma albicincta (Hampson, 1905)

116. Epidesma aurimacula (Schaus, 1905)

117. Epidesma hoffmannsi (Rothschild, 1912) AMZ $^{\mathrm{A}}$

118. Epidesma klagesi (Rothschild, 1912)

119. Epidesma lenaeus (Cramer, 1780)

120. Epidesma metapolia (Dognin, 1912)

121. Epidesma parva (Rothschild, 1912)

\section{Record}

AM, Fonte Boa (Rothschild 1912)

RO, [Porto Velho] \{Madeira river, Aliança\} (Rothschild 1912)

AM, [Atalaia do Norte] \{Rio Javary, Braga\} (Butler 1878), São Paulo de Olivença*; AP, Serra do Navio*; PA, Marabá*, Santarém \{Taperinha\} (Zerny 1931); RO, Cacaulândia*, Porto Velho*

AM, Fonte Boa (Rothschild 1912); PA, Santarém \{Taperinha\} (Zerny 1931); RO, Ariquemes*, Porto Velho*

[AM] (Hampson 1901); PA (Hagmann 1938)

AC, Feijó \{Rio Embira\} (Travassos Filho \& Urban 1967); AM, Benjamin Constant*, Fonte Boa*, Humaitá*, [Manaus] \{Rio Urubu\}*, Manicoré*, São Paulo de Olivença \{Rio Preto\} (Travassos Filho 1957), Tabatinga*; MA, Açailândia*; MT, Aripuanã*; PA, Altamira \{Monte Santo\} (Teston \& Delfina 2010), Belém*, Capitão Poço*, Parauapebas \{Serra Norte, Carajás $\}^{*}$, Tucuruí*, Viseu*; RO, Porto Velho \{Rio Madeira\} (Travassos Filho \& Urban 1967)

AM, Benjamin Constant*; MA, Açailândia*

[AM], Amazonas river (Rothschild 1911); RO, [Porto Velho] \{Madeira river, Calama\} (Rothschild 1911)

PA, Santarém \{Taperinha\} (Zerny 1931)

PA, Santarém*

AM, [Jutaí] \{Rio Jutaí\} (Butler 1878), Manaus*, Santa Isabel do Rio Negro*; AP, Serra do Navio*; MA, Açailândia*; PA, Cametá (Herrich-Schäffer [1855]), Altamira $\left\{51^{\circ} \mathrm{BIS}\right\}$ (Teston et al. 2012), Belém*, Belterra \{National Forest of Tapajós\} (Freitas 2014), Santarém \{Taperinha\} (Zerny 1931)

PA, Santarém \{Taperinha\} (Zerny 1931)

PA, Santarém*

AM, Humaitá \{Madeira river\} (Rothschild 1911)

AM, [Carauari] \{Rio Juruá, Pupunhazinho\} (Butler 1878), Manicoré \{Madeira river\} (Zerny 1931), Tefé \{Ega\} (Butler 1876); MA, Açailândia*, PA, Marabá*, Novo Progresso $\left\{\right.$ Cachimbo ${ }^{*}$, Oriximiná \{Rio Cuminá\}*, Rio Tapajós (Butler 1876), Santarém (Walker 1854a), Santarém \{Taperinha\} (Zerny 1931), São Félix do Xingu \{Serra do Pardo National Park\} (Teston \& Correa 2015); RO, Cacaulândia*, Porto Velho*

PA (Hagmann 1938), Altamira \{Monte Santo\} (Delfina \& Teston 2013), Santarém*

AM, São Paulo de Olivença*; PA (Hagmann 1938), Altamira \{Serra do Pardo National Park\} (Teston \& Correa 2015), Marabá*, Santarém*, São Félix do Xingu \{Serra do Pardo National Park\} (Teston \& Correa 2015)

RO, Cacaulândia*

AM, Fonte Boa (Rothschild 1912); PA, Santarém \{Taperinha\} (Zerny 1931); RO, [Porto Velho] \{Madeira river, Calama\} (Rothschild 1912)

AM, Tefé (Zerny 1931); MA, Açailândia*; PA, Altamira \{Serra do Pardo National Park\} (Teston \& Correa 2015), Belém*, Marabá*, Santarém \{Taperinha\} (Zerny 1931), São Félix do Xingu \{Serra do Pardo National Park\} (Teston \& Correa 2015); RO, Cacaulândia*

PA, Marabá*; RO, Cacaulândia*, Jarú*, Porto Velho*

PA, Altamira \{Monte Santo\} (Delfina \& Teston 2013), Marabá*, Santarém*, São Félix do Xingu \{Serra do Pardo National Park\} (Teston \& Correa 2015); RO, Porto Velho* 
Teston, J.A. et al.

Continuation Table 1.

\begin{tabular}{ll}
\hline & Species \\
\hline 122. & Epidesma perplexa (Rothschild, 1912) \\
123. & Epidesma similis (Rothschild, 1912) \\
124. & Epidesma ursula (Cramer, 1782)
\end{tabular}

125. Episcepsis capyscoides Dognin, 1911 ${ }^{\mathrm{NEW}}$

126. Episcepsis endodasia (Hampson, 1898)

127. Episcepsis frances Dyar, 1910

128. Episcepsis gnoma (Butler, 1877)

129. Episcepsis gnomoides Schaus, 1910

130. Episcepsis klagesi Rothschild, 1911

131. Episcepsis lamia (Butler, 1877)

132. Episcepsis littoralis Rothschild, 1911

133. Episcepsis melanitis (Hübner, 1827)

134. Episcepsis nereus Zerny, 1931

135. Episcepsis luctuosa (Möschler, 1877)

136. Episcepsis thetis (Linnaeus, 1771)

137. Episcepsis venata Butler, 1877

138. Eriphioides tractipennis (Butler, 1876) NEW $^{\mathrm{N}}$

139. Euagra coelestina (Cramer, 1782)

140. Euagra intercisa Butler, $1876^{\mathrm{AMZ}}$

141. Eucereon aeolum Hampson, $1898^{\mathrm{AMZ}}$

Record

AM, [Atalaia do Norte] \{Santo Antonio do Javari\} (Rothschild 1912), Fonte Boa (Rothschild 1912), Tefé (Rothschild 1912)

AM, Fonte Boa (Rothschild 1912)

AM, [Jutaí] \{Rio Jutaí\} (Butler 1878), [Lábrea] \{Rio Purus, Man[aua]\} (Butler 1878), Manaus*, Rio Sapo (Butler 1878), [São Gabriel da Cachoeira] \{Taracuá\} (Bryk 1953), São Paulo de Olivença*, Tefé \{Ega\} (Hampson 1898); MA, Açailândia*; PA, Almeirim \{Jari\} (Hawes et al. 2009), Altamira \{Serra do Pardo National Park\} (Teston \& Correa 2015), Belterra \{National Forest of Tapajós\} (Freitas 2014), Cametá*, Marabá*, Novo Progresso \{Cachimbo\}*, Santarém \{Taperinha\} (Zerny 1931), São Félix do Xingu \{Serra do Pardo National Park\} (Teston \& Correa 2015), Tapajós [river] (Hampson 1898); RO, Cacaulândia*, Porto Velho*; [RR], [Caracaraí] \{Branco river\} (Bryk 1953)

RO, Cacaulândia*

AM, Borba*; PA, São Félix do Xingu \{Serra do Pardo National Park\} (Teston \& Correa 2015)

PA, Altamira $\left\{51^{\circ} \mathrm{BIS}\right\}$ (Teston et al. 2012), Breves*, Santarém \{Taperinha\} (Zerny 1931)

AM, [Barcelos] \{Rio Pada[ua]r[i]\} (Butler 1877), Santa Isabel do Rio Negro*, São Paulo de Olivença*; PA, Belém*, Breves (Zerny 1931), Santarém \{Taperinha\} (Zerny 1931), São Félix do Xingu \{Serra do Pardo National Park\} (Teston \& Correa 2015)

PA, Santarém \{Taperinha\} (Zerny 1931)

AM, São Paulo de Olivença*; PA, Belterra \{National Forest of Tapajós\} (Freitas 2014), Breves*, Santarém \{Taperinha\} (Zerny 1931); RO, Cacaulândia*

AM, Benjamin Constant*, Borba*, [Maués] \{Rio Maués\} (Butler 1877), [Tapauá] \{Rio Purus, Ta[u]aria\} (Butler 1877); MA, Açailândia*; PA, Belém*, Belterra \{National Forest of Tapajós\} (Freitas 2014), Breves*, Santarém \{Taperinha\} (Zerny 1931), São Félix do Xingu \{Serra do Pardo National Park\} (Teston \& Correa 2015); RO, Ariquemes*

AM, [São Gabriel da Cachoeira] \{Taracuá\} (Bryk 1953)

AM, Manaus*, Manicoré*, Santa Isabel do Rio Negro*, [São Gabriel da Cachoeira] \{Taracuá\} (Bryk 1953), Tefé*; MT, Sinop*; PA, [Belém] (Hagmann 1938)

AM, São Paulo de Olivença*; PA, Belém*, Santarém \{Taperinha\} (Zerny 1931)

AM, Fonte Boa (Rothschild 1911); PA, Breves*, Santarém \{Taperinha\} (Zerny 1931), São Félix do Xingu \{Serra do Pardo National Park\} (Teston \& Correa 2015); RO, Cacaulândia*, [Porto Velho] \{Madeira river, Aliança and Calama\} (Rothschild 1911)

MT, Sinop*; PA, Santarém*, São Félix do Xingu \{Serra do Pardo National Park\} (Teston \& Correa 2015)

AM, [Jutaí] \{Rio Jutaí near Rio Cur[uena]\} (Butler 1877); PA, Belém*, Belterra \{National Forest of Tapajós\} (Freitas 2014), Marabá*, Prainha to Monte Alegre (Zerny 1931), Santarém \{Taperinha\} (Zerny 1931), São Félix do Xingu \{Serra do Pardo National Park\} (Teston \& Correa 2015); RO, Ariquemes*, Cacaulândia*

PA, Santarém*

AM, Santa Isabel do Rio Negro*; PA, [Belém] (Walker 1854b), Santarém*

PA, Anajás*, Belém*

MA, Açailândia* 
Continuation Table 1.

\begin{tabular}{ll} 
Species \\
\hline 142. & Eucereon amazonum (Rothschild, 1912)
\end{tabular}

143. Eucereon aoris Möschler, 1877

144. Eucereon archias (Stoll, 1790)

145. Eucereon arenosa Butler, 1877

146. Eucereon atriguttum Druce, 1905

147. Eucereon brunnea (Hampson, 1903)

148. Eucereon complicatum Butler, 1877

149. Eucereon confinis Herrich-Schäffer, 1855

150. Eucereon dorsipuncta Hampson, 1905 AMZ

151. Eucereon exprata Dognin, 1921

152. Eucereon fuscoirroratum (Rothschild, 1912)

153. Eucereon hagmanni (Travassos, 1952)

154. Eucereon hoffmansi (Rothschild, 1912)

155. Eucereon latifascia (Walker, 1856)

156. Eucereon maia Druce, 1884

157. Eucereon marmoratum Butler, 1877

158. Eucereon melanoperas Hampson, 1898

159. Eucereon metoidesis Hampson, 1905

160. Eucereon mitigatum Walker, 1857

\section{Record}

AM, Fonte Boa (Rothschild 1912), São Paulo de Olivença*; PA, Breves*, Santarém \{Taperinha\} (Zerny 1931), São Félix do Xingu \{Serra do Pardo National Park\} (Teston \& Correa 2015)

AM, Manicoré*; AP, Serra do Navio*; MT, Sinop*; PA, Altamira \{Monte Santo\} (Teston \& Delfina 2010), Belterra \{National Forest of Tapajós\} (Freitas 2014), Santarém \{Taperinha\} (Zerny 1931)

AM, Manicoré \{Rio Madeira\} (Travassos 1959), Rio Javari (Butler 1878), Rio Japura (Pinheiro 2016), Tefé (Zerny 1931), Purus river (Bryk 1953); PA, Ale[n] quer (Pinheiro 2016), Gurupá to Almeirim (Zerny 1931), [Breves] \{Furos de Breves\} (Zerny 1931), Santarém \{Taperinha\} (Zerny 1931)

AM, São Paulo de Olivença*; PA, Santarém*; RO, [Porto Velho] \{Rio Madeira, Jam[a]ry\} (Butler 1877)

AP, Serra do Navio*; PA, Marabá*, Santarém*, São Félix do Xingu \{Serra do Pardo National Park $\}$ (Teston \& Correa 2015), São Miguel do Guamá*; RO, Cacaulândia*

PA, Santarém \{Taperinha\} (Zerny 1931); RO, [Porto Velho] \{Madeira river, Aliança\} (Rothschild 1912)

AM, Manicoré \{Rio Madeira\} (Zerny 1931); Rio Juruá (Butler 1877); PA, Santarém \{Taperinha\} (Zerny 1931), São Félix do Xingu \{Serra do Pardo National Park (Teston \& Correa 2015); RO, Cacaulândia*

[AM], Vale do [Rio] Amazonas (Travassos 1952)

RO, Cacaulândia*

PA, [Igarapé Açu] \{Prata 100 km of Pará [Belém]\} (Dognin 1921)

PA, Santarém \{Taperinha\} (Zerny 1931)

AM, Borba \{Lago Acará\} (Travassos 1952), São Paulo de Olivença (Travassos 1952); PA, Belém \{Bosque\} (Travassos 1952), Santarém*

RO, [Porto Velho] \{Aliança\} (Rothschild 1912)

AM, [Autazes] \{Autaz river\} (Bryk 1953); MA, Açailândia*; PA, [Belém] (Walker 1856), Altamira \{Monte Santo\} (Teston \& Delfina 2010), Marabá*, Santarém \{Taperinha\} (Zerny 1931), São Félix do Xingu \{Serra do Pardo National Park\} (Teston \& Correa 2015), [Novo Progresso] \{Cachimbo\} (Travassos 1964a), Óbidos (Zerny 1931); RO, Cacaulândia*

AM, Tefé*; PA, Altamira \{Monte Santo\} (Teston \& Delfina 2010), Belém*, Santarém \{Taperinha\} (Zerny 1931); RO, Cacaulândia*

AM, [Carauari] \{Rio Juruá, Curimatá and [Lake of] Pupunha) (Butler 1877), Beruri \{Rio Purus, Aruman\} (Butler 1877), [Lábrea] \{Rio Purus, Mabid[e]r[i]\} (Butler 1877), [Novo Airão] \{Rio Negro, Ayrão\} (Butler 1877), [Santa Isabel do Rio Negro] \{Rio Preto\}*, São Paulo de Olivença*; PA, Breves*, Santarém \{Taperinha\} (Zerny 1931), São Félix do Xingu \{Serra do Pardo National Park\} (Teston \& Correa 2015), [Oriximiná] \{Rio Trombetas, Cachoeira [da] Porteira\} (Butler 1877); RO, Porto Velho*

AM, São Paulo de Olivença*, Purus river (Hampson 1898), Tefé \{Ega $\}$ (Hampson 1898); PA, Santarém \{Taperinha\} (Zerny 1931)

AM, [São Gabriel da Cachoeira] \{Taracuá\} (Bryk 1953); AP, Serra do Navio*; PA, [Belém] (Butler 1876), Belterra \{National Forest of Tapajós\} (Freitas 2014), Santarém \{Taperinha\} (Zerny 1931)

AM, Benjamin Constant \{Rio Javary, Alto Amazonas\} (Pinheiro 2016), Fonte Boa (Pinheiro 2016), [Jutaí] \{Rio Jutaí, Boa Vista\} (Butler 1877), [Lábrea] \{Rio Purus, Huitanaã\} (Butler 1878), [Novo Airão] \{Igarapé Preto, Alto Amazonas\} (Pinheiro 2016), São Paulo de Olivença (Pinheiro 2016); PA, [Belém] (Pinheiro 2016) Chaves \{Marajó Island\} (Hampson 1898), Santarém \{Taperinha\} (Pinheiro 2016) 
Teston, J.A. et al.

Continuation Table 1.

\begin{tabular}{ll}
\hline \multicolumn{2}{l}{ Species } \\
\hline 161. & Eucereon obscura (Möschler, 1872) \\
& \\
& \\
162. & Eucereon parascyton Hampson, 1914 \\
163. & Eucereon patrona Schaus, $1896^{\mathrm{AMZ}}$ \\
164. & Eucereon plumbicollum Hampson, $1898^{\mathrm{NEW}}$ \\
165. & Eucereon pseudarchias Hampson, 1898
\end{tabular}

166. Eucereon punctatum (Guérin-Méneville, [1844])

167. Eucereon reniferum Hampson, $1898^{\text {NEW }}$

168. Eucereon rosa (Walker, 1854)

169. Eucereon rosina (Walker, 1854) ${ }^{\text {NEW }}$

170. Eucereon scyton (Cramer, 1777)

171. Eucereon setosa (Sepp, [1830]) $)^{\mathrm{AMZ}}$

172. Eucereon simile (Draudt, 1915)

173. Eucereon sylvius (Stoll, 1790)

174. Eucereon taperinhae Dognin, 1923

175. Eucereon tarona Hampson, 1898

176. Eucereon varia (Walker, 1854)

177. Eucereon velutina Schaus, $1896^{\mathrm{NEW}}$

178. Euceriodes wernickei (Draudt, 1917)

179. Eumenogaster affinis Rothschild, 1911 NEW

180. Eumenogaster haemacera Hampson, $1898^{\mathrm{NEW}}$

181. Eumenogaster nigricauda Dognin, 1911

182. Eumenogaster notabilis (Walker, [1865])

\section{Record}

AM, Benjamin Constant*, Fonte Boa*, [Jutaí] \{Rio Jutaí\} (Butler 1877), Manicoré*, São Paulo de Olivença*; AP, Serra do Navio*; MA, Açailândia*; PA, Altamira $\left\{51^{\circ} \mathrm{BIS}\right\}$ (Teston et al. 2012), Belém*, Belterra \{National Forest of Tapajós\} (Freitas 2014), Breves*, Santarém \{Taperinha\} (Zerny 1931), Viseu*; RO, Porto Velho*

AM, São Paulo de Olivença*; PA, Santarém \{Taperinha\} (Zerny 1931)

RO, Cacaulândia*

AP, Serra do Navio*

AM, Juruá river (Hampson 1898); PA, Altamira \{Serra do Pardo National Park\} (Teston \& Correa 2015), [Belém] (Travassos 1964a), Belém \{Miramar\}*, Santarém \{Taperinha\} (Zerny 1931), São Félix do Xingu \{Serra do Pardo National Park\} (Teston \& Correa 2015); RO, Cacaulândia*

AM, Benjamin Constant*, [Eirunepé] \{Rio Juruá, São Felipe\} (Zerny 1931), São Paulo de Olivença*, Tefé*; PA, Breves*, Santarém \{Taperinha\} (Zerny 1931); RO, Cacaulândia (Pinheiro 2016)

RO, Cacaulândia*

AM, Beruri \{Rio Purus, Paricatuba\} (Butler 1878)

MA, Açailândia*; RO, Cacaulândia*

AM, Benjamin Constant*; PA, Marabá*, Santarém \{Taperinha\} (Zerny 1931); RO, Ariquemes*, Cacaulândia*, Jarú*, Porto Velho*

MA, Açailândia*; PA, Novo Progresso $\{\text { Cachimbo }\}^{*}$

AM, Manicoré (Zerny 1931); PA, Santarém \{Taperinha\} (Zerny 1931); RO, [Porto Velho] \{Aliança\} (Rothschild 1912)

[AC], Upper Juruá river (Zerny 1931); AM, [Jutaí] \{Rio Jutaí, Barreira Branca\} (Butler 1878), [Eirunepé] \{Rio Juruá, São Felipe\} (Zerny 1931), Rio Juruá \{Pupunhazinho\} (Butler 1878), Rio Negro (Travassos 1959), São Paulo de Olivença \{Rio Preto (Travassos 1959), Tefé (Zerny 1931); PA, [Belém] “Amazonas inferior" (Travassos 1959), Breves*, Santarém (Hampson 1898), Santarém \{Taperinha\} (Zerny 1931), São Félix do Xingu \{Serra do Pardo National Park $\}$ (Teston \& Correa 2015)

PA, Santarém \{Taperinha\} (Dognin 1923)

PA, Marabá*, São Félix do Xingu \{Serra do Pardo National Park\} (Teston \& Correa 2015); RO, Cacaulândia*

AM, [Atalaia do Norte] \{Rio Quichito\} (Travassos 1964a), Benjamin Constant*, Borba \{Rio Madeira e Lago Acar[á]\} (Travassos 1964a), [Canutama] \{Rio Purus, Urucuri\} (Butler 1878), [Carauari] \{Rio Juruá, [Lake of] Pupunha\} (Butler 1878), [Eirunepé] \{Juruá river, São Felipe\} (Zerny 1931), Manaus (Travassos 1964a), Manicoré \{Madeira river\} (Zerny 1931), [Maués] \{Rio Maués\} (Travassos 1964a), São Paulo de Olivença*, Rio Preto (Travassos 1964a), Rio Pauary (Travassos 1964a); MT, Sinop*; PA, Altamira \{Serra do Pardo National Park\} (Teston \& Correa 2015), [Belém] (Walker 1854a), Breves*, Gurupá-Almeirim (Zerny 1931), Marabá*, [Novo Progresso] \{Cachimbo\} (Travassos 1964a), Santarém \{Taperinha\} (Zerny 1931), São Félix do Xingu \{Serra do Pardo National Park\} (Teston \& Correa 2015); RO, Cacaulândia*

RO, Cacaulândia*

PA, [Novo Progresso] \{Cachimbo $\}$ (Travassos 1964a)

PA, Belém*

AM, Manicoré*

PA, Belém*

AM, Manaus \{Pensador\} (Bryk 1953); PA, Belém*, Santarém \{Taperinha\} (Zerny 1931), Tapajós [river] (Walker [1865]) 
Continuation Table 1.

\begin{tabular}{ll}
\hline \multicolumn{2}{l}{ Species } \\
\hline 183. & Eumenogaster psedosphecia Hampson, 1898 \\
184. & Heliura amazonicum (Rothschild, 1912) \\
185. & Heliura balia (Hampson, 1898) \\
186. & Heliura cadroe Schaus, 1924 \\
187. & Heliura excavata (Dognin, 1910) \\
188. & Heliura hagmanni Zerny, 1931 \\
189. & Heliura marica (Cramer, 1775)
\end{tabular}

190. Heliura perexcavatum (Rothschild, 1912)

191. Heliura phaeosoma Druce, 1905

192. Heliura pierus (Cramer, 1782)

193. Heliura postcoeruleum (Rothschild, 1912)

194. Heliura rhodophila (Walker, 1856)

195. Heliura semihyalina (Rothschild, 1912)

196. Heliura stolli Rothschild, 1912

197. Heliura suffusa (Lathy, 1899)

198. Heliura tetragramma (Walker, 1854)

199. Heliura thysbodes Dognin, 1914

200. Heliura zonata Druce, 1905

201. Herea metaxanthus (Walker, 1854)

202. Herea ruficeps (Walker, 1854)

203. Hyaleucerea erythotela (Walker, 1854)

204. Hyaleucerea fusiformis (Walker, 1856)

205. Hyaleucerea lemoulti Schaus, 1905

206. Hyaleucerea leucosticta Druce, 1905

\section{Record}

AM, Manaus (Bryk 1953), Tefé (Zerny 1931), Tonantins (Hampson 1898); PA, Belém*, Santarém \{Taperinha\} (Zerny 1931)

AM, Fonte Boa (Rothschild 1912)

PA, Altamira \{Monte Santo\} (Teston \& Delfina 2010), São Félix do Xingu \{Serra do Pardo National Park $\}$ (Teston \& Correa 2015)

MA, Açailândia*; PA, Belém*, Capitão Poço*; RO, Cacaulândia*

AM, Benjamin Constant*; PA, Belterra \{National Forest of Tapajós\} (Freitas 2014), Igarapé Açú*, Santarém (Valente et al. 2018)

AM, Manicoré*; PA, Santarém \{Taperinha\} (Zerny 1931)

AM, Borba*, [Lábrea] \{Purus river, [S]epatiny\} (Hampson 1898), São Gabriel da Cachoeira \{Querari\}*, São Paulo de Olivença*, Tefé*; AP, Serra do Navio*; MT Sinop*; PA, [Belém] (Walker 1854a), Breves*, Santarém \{Taperinha\} (Zerny 1931)

AM, Borba*, Fonte Boa (Rothschild 1912); PA, Belterra \{National Forest of Tapajós\} (Freitas 2014)

AM, Manicoré*, Santa Isabel do Rio Negro*; PA, Belém \{Miramar\}*, Breves*, Marabá*, Santarém \{Taperinha\} (Zerny 1931); RO, Cacaulândia*

AM, Fonte Boa*, [Jutaí] \{Rio Jutaí\} (Butler 1878); AP, Porto Grande*, Serra do Navio*; PA, Belém*; RO, Cacaulândia*, Porto Velho*

AM, Manicoré (Zerny 1931), Tefé (Zerny 1931); PA, [Belém] (Hampson 1914), Santarém \{Taperinha\} (Zerny 1931); RO, [Porto Velho] \{Rio Madeira, Aliança and Calama $\}$ (Rothschild 1912)

AM, Manicoré*; MA, Açailândia*; PA, [Belém] (Butler 1876), Altamira \{Monte Santo\} (Teston \& Delfina 2010), Marabá*, Santarém \{Taperinha\} (Zerny 1931); RO, Ariquemes*

AM, Fonte Boa (Rothschild 1912)

AM, Benjamin Constant*, Fonte Boa (Rothschild 1912), Tefé (Rothschild 1912)

PA, Santarém \{Taperinha\} (Zerny 1931), São Félix do Xingu \{Serra do Pardo National Park\} (Teston \& Correa 2015); RO, Cacaulândia*

MA, Açailândia*; MT, Sinop*; PA, Belterra \{National Forest of Tapajós\} (Freitas 2014), Santarém (Walker 1854a), Santarém \{Taperinha\} (Zerny 1931), São Félix do Xingu \{Serra do Pardo National Park\} (Teston \& Correa 2015)

AM, São Paulo de Olivença (Dognin 1923)

AM, Benjamin Constant*, Fonte Boa*, Manaus*; MA, Açailândia*; MT, Sinop*; PA, Óbidos*, Santarém \{Taperinha\} (Zerny 1931), São Félix do Xingu \{Serra do Pardo National Park $\}$ (Teston \& Correa 2015); RO, Cacaulândia*

PA, [Belém] (Walker 1854a)

PA, [Belém] (Walker 1854a), Santarém \{Taperinha\} (Zerny 1931), Tapajós [river] (Hampson 1898)

PA, Altamira \{Serra do Pardo National Park\} (Teston \& Correa 2015), [Belém] (Walker 1854a), Santarém \{Taperinha\} (Zerny 1931), São Félix do Xingu \{Serra do Pardo National Park $\}$ (Teston \& Correa 2015)

AM, Valley of Amazonas [river] (Walker 1856), São Gabriel [da Cachoeira] \{Taracuá\} (Bryk 1953); PA, Belterra \{National Forest of Tapajós\} (Freitas 2014), Santarém \{Taperinha\} (Zerny 1931), São Félix do Xingu \{Serra do Pardo National Park\} (Teston \& Correa 2015)

PA, Belterra \{National Forest of Tapajós\} (Freitas 2014), Santarém \{Taperinha\} (Zerny 1931)

PA, Belterra \{National Forest of Tapajós\} (Freitas 2014), Santarém \{Taperinha\} (Zerny 1931), São Félix do Xingu \{Serra do Pardo National Park\} (Teston \& Correa 2015) 
Teston, J.A. et al.

Continuation Table 1.

\begin{tabular}{lll}
\hline \multicolumn{1}{c}{ Species } & Record \\
\hline 207. $\begin{array}{l}\text { Hyaleucerea manicorensis Rêgo Barros \& } \\
\text { Machado Filho, 1971 }\end{array}$ & AM, Manicoré (Rêgo Barros \& Machado Filho 1971) \\
208. Hyaleucerea trifasciata (Butler, 1877) & $\begin{array}{l}\text { AM, [Lábrea] \{Rio Purus, Jurucuá\} (Butler 1877), Solimões [river] (Hampson } \\
\text { 1898) }\end{array}$ \\
209. Hyaleucerea vulnerata Butler, 1875 & AM, São Paulo de Olivença (Rêgo Barros \& Machado Filho 1971); AP, Serra \\
& do Navio (Rêgo Barros \& Machado Filho 1971); MT, Sinop*; PA (Travassos
\end{tabular}

210. Hypocladia elongata Druce, 1905

AM, Benjamin Constant*, Manaus (Zerny 1931), Tefé*; AP, Serra do Navio*; PA, [Igarapé-Miri] \{estuary of Tocantins [river]\} (Zerny 1931), Santarém \{Taperinha\} (Zerny 1931)

211. Hypocladia militaris (Butler, 1877)

212. Hypocladia parcipuncta Hampson, 1909

AM, Silves (Butler 1877); RO, Cacaulândia*

PA, [Breves] \{Furos de Breves\} (Zerny 1931)

213. Loxozona lanceolata (Walker, 1854)

214. Lymire fulvicollis Dognin, 1914

215. Lymire metamelas (Walker, 1854)

216. Lymire nitens (Rothschild, 1912) LEW $^{\mathrm{NE}}$

217. Lymire strigivenia Druce, 1905

218. Metastatia pyrrhorhoea (Hübner, 1827)

219. Paraethria angustipennis (Rothschild, 1911)

220. Paraethria mapiria Draudt, 1915

221. Patreliura capys (Cramer, 1775)

PA, [Belém] (Walker 1854a), São Félix do Xingu \{Serra do Pardo National Park\} (Teston \& Correa 2015)

AM, São Gabriel [da Cachoeira] (Bryk 1953)

AM, Santa Isabel do Rio Negro*; AP, Serra do Navio*; PA, [Belém] (Walker 1854a), Breves*, Santarém \{Taperinha\} (Zerny 1931); RO, Porto Velho*

PA, Belém*

PA, Belterra \{National Forest of Tapajós\} (Freitas 2014)

AM, Manicoré*, [Maués] \{Rio Parauary\}*, Santa Isabel do Rio Negro*, Tefé*; PA, Anajás*, [Belém] (Hampson 1898), Capitão Poço*, Óbidos*, Ourém*, Santarém \{Taperinha\} (Zerny 1931)

PA, Itaituba to Óbidos (Rothschild 1911)

PA, [Belém](Hagmann 1938), Belterra \{National Forest of Tapajós\} (Freitas 2014)

AM, Beruri \{Rio Purus, Aruman\} (Zerny 1931), Borba*, [Lábrea] \{Rio Purus, Jurucuá\} (Zerny 1931), Rio Juruá (Hampson 1898), [Santa Isabel do Rio Negro] \{Rio Preto $\}^{*}$, [São Gabriel da Cachoeira] \{Taracuá\} (Bryk 1953), São Paulo de Olivença*, Tefé \{Ega\} (Butler 1876); PA, Marabá*, Santarém \{Taperinha\} (Zerny 1931), [Rio] Tapajós (Butler 1876); RO, Porto Velho*; [RR], [Caracaraí] \{Rio Branc[o]\} (Bryk 1953)

222. Philoros rubriceps (Walker, 1854)

223. Pseudaclytia opponens (Walker, [1865])

[AM], (Zerny 1912); PA, Belém*

AM, [Autazes] \{Rio Autaz\} (Bryk 1953), Barcelos \{Rio Negro, Carvoeiro\} (Butler 1878), Borba*, Manicoré*, [Maués] \{Rio Maués\} (Butler 1878), Rio Juruá (Butler 1878), Rio Negro (Butler 1878), Rio Purus (Bryk 1953), São Gabriel [da Cachoeira] (Bryk 1953), Tefé \{Ega\}(Walker [1865]), Tonantins (Butler 1878); AP, Serra do Navio*; PA, Breves*, Santarém \{Taperinha\} (Zerny 1931); RO, Cacaulândia*, Porto Velho*; [RR], [Caracaraí] \{Rio Branco\} (Bryk 1953)

AM, Fonte Boa*

PA, Belterra \{National Forest of Tapajós\} (Freitas 2014)

AM, Manaus (Bryk 1953)

[AM], Amazonas [river] (Druce 1898); AM, Fonte Boa*, Manicoré*, Tefé*; PA, Belém*, Novo Progresso \{Cachimbo\}*, Parauapebas \{Serra Norte, Carajás\}*, Santarém (Valente et al. 2018), São Félix do Xingu \{Serra do Pardo National Park\} (Teston \& Correa 2015); RO, Cacaulândia*, Jarú*

228. Pseudosphenoptera basalis (Walker, 1854) 
Continuation Table 1.

\begin{tabular}{|c|c|}
\hline & Species \\
\hline 229. & Pseudosphenoptera boyi Zerny, 1931 \\
\hline 230. & Pseudosphex aracia D. Jones, 1914 ${ }^{\mathrm{NEW}}$ \\
\hline 231. & Pseudosphex caurensis Klages, 1906 \\
\hline 232. & $\begin{array}{l}\text { Pseudosphex ichneumonea Herrich-Schäffer, } \\
1854^{\mathrm{Amz}}\end{array}$ \\
\hline 233. & Pseudosphex klagesi Rothschild, 1911 \\
\hline 234. & Pseudosphex polistes Hübner, 1827 \\
\hline 235 . & Pseudosphex rubripalpus Hampson, 1901 ${ }^{\mathrm{AMZ}}$ \\
\hline 236. & Ptychotricos fenestrifer Zerny, 1931 \\
\hline 237. & Ptychotricos zeus Schaus, 1894 \\
\hline
\end{tabular}

238. Riccia aliaria (Druce, 1890)

239. Schasiura mimica Butler, 1877

240. Sciopsyche tropica (Walker, 1854)

241. Sesiura smaragdina (Walker, [1865])

242. Syntrichura sphecomorpha Bryk, 1953

243. Syntrichura virens Butler, $1876^{\mathrm{NEW}}$

244. Telioneura albapese (Druce, 1898)

245. Telioneura brevipennis (Butler, 1877)

246. Telioneura fuliginosa (Rothschild, 1910)

247. Telioneura glaucopis R. Felder, 1869

248. Telioneura hypophaeus (Hampson, 1905)

249. Theages albidias (Rothschild, 1912)

250. Theages leucophaea Walker, 1855

251. Theages xanthura (Schaus, 1910) ${ }^{\mathrm{AMZ}}$

252. Timalus leucomela (Walker, 1856)

\section{Record}

PA, Santarém \{Mojú\} (Zerny 1931)

PA, Belém*, Breves*, Santarém*

PA, [Belém] (Rothschild 1931)

AM, Santa Isabel do Rio Negro*; PA, Novo Progresso $\{$ Cachimbo\}*

PA [Belém] (Hagmann 1938), Santarém*

AM, Tefé \{Ega\} (Walker 1854a); PA, Belém*, Capitão Poço*, Santarém \{Taperinha\} (Zerny 1931); RO, [Porto Velho] \{Rio Machados\} (Zerny 1931)

AM, Manicoré*; MT, Sinop*

PA, Santarém \{Taperinha\} (Zerny 1931)

MA, Açailândia*; MT, Sinop*; PA, Altamira \{Serra do Pardo National Park\} (Teston \& Correa 2015), Novo Progresso \{Cachimbo $\}^{*}$, Parauapebas $\{$ Serra Norte, Carajás $\}^{*}$, Santarém \{Taperinha\} (Zerny 1931), São Félix do Xingu \{Serra do Pardo National Park $\}$ (Teston \& Correa 2015)

AM, Borba*, São Paulo de Olivença (Travassos Filho 1953)

AM, Rio Solimões \{Barreira das Araras\} (Butler 1877); MT, Sinop*; PA, Novo Progresso $\{\text { Cachimbo }\}^{*}$; RO, Cacaulândia*

AM, Tefé (Butler 1876); PA, Santarém (Butler 1876); RO, Cacaulândia*, Jarú* AM, [Autazes] \{Rio Autaz\} (Bryk 1953), Manicoré*, Tefé \{Ega\} (Walker [1865]); PA, Belém*, Santarém*

AM, [São Gabriel da Cachoeira] \{Taracuá\} (Bryk 1953)

AM, São Paulo de Olivença*; PA, Belém*, Santarém*

AM, Manicoré*; MT, Sinop*; PA, Belém*, Santarém (Valente et al. 2018)

AM, Manicoré*, [Tapauá] \{Rio Purus, Guajaratuba\} (Butler 1877); MA, Açailândia*; PA, Altamira \{Monte Santo\} (Delfina \& Teston 2013), Belém*; RO, Porto Velho*

AM, Fonte Boa (Rothschild 1910)

AM, Barcelos \{Moura\}*, Beruri \{Rio Purus, Aruman\} (Butler 1878), [Canutama] \{Rio Purus, Urucuri\} (Butler 1878), [Carauari] \{Rio Juruá, Curimatá\} (Butler 1878), [Eirunepé] \{Rio Juruá, São Felipe\} (Zerny 1931), Fonte Boa*, [Lábrea] \{Rio Purus, Jurucuá e Man[aua]\} (Butler 1878), Manicoré*, [Maués] \{Rio Parauary $\}^{*}$, Amazonas [river] (R. Felder 1869), Rio Negro (Butler 1878), São Paulo de Olivença*, Tefé*; AP, Serra do Navio*; PA, Altamira \{Serra do Pardo National Park\} (Teston \& Correa 2015), Parauapebas \{Serra Norte, Carajás $\}$ *, Santarém \{Taperinha\} (Zerny 1931); RO, Cacaulândia*, Candeias do Jamari*, Jarú*, Porto Velho*

PA, Santarém \{Taperinha\} (Zerny 1931)

AM, Fonte Boa (Hampson 1914), Manaus (Hampson 1914); PA, Marabá*, [Novo Progresso] \{Cachimbo\} (Travassos 1964b), Santarém \{Taperinha\} (Zerny 1931); RO, Cacaulândia*, Porto Velho*

PA, São Félix do Xingu \{Serra do Pardo National Park\} (Teston \& Correa 2015)

AM, Benjamin Constant*; MT, Sinop*; PA, Santarém*; RO, Cacaulândia*, Candeias do Jamari*

AM, Manicoré*; MT, Sinop*; PA, [Belém] (Walker 1856), Novo Progresso $\{\text { Cachimbo }\}^{*}$, Parauapebas $\{\text { Serra Norte, Carajás }\}^{*}$, Santarém $\{$ Mojú\} and \{Taperinha\} (Zerny 1931), São Félix do Xingu \{Serra do Pardo National Park\} (Teston \& Correa 2015); RO, Cacaulândia* 
Teston, J.A. et al.

Continuation Table 1.

\begin{tabular}{|c|c|c|}
\hline & Species & Record \\
\hline 253. & Trichura aurifera Butler, 1876 & $\begin{array}{l}\text { AM, Santa Isabel do Rio Negro*; AP, Serra do Navio*; MA, Santa Luzia*; PA, } \\
\text { [Belém] (Butler 1876), Altamira \{Serra do Pardo National Park\} (Teston \& Correa } \\
\text { 2015), Santarém \{Taperinha\} (Zerny 1931) }\end{array}$ \\
\hline 254. & Trichura cerberus (Pallas, 1772) & $\begin{array}{l}\text { AM, Manaus*, Upper Amazon (Zerny 1912), São Paulo de Olivença*, Santa } \\
\text { Isabel do Rio Negro*; PA, Belém*, Breves*, Capitão Poço*, Óbidos*, Santarém } \\
\text { \{Taperinha\} (Zerny 1931); RO, Porto Velho* }\end{array}$ \\
\hline 256. & Trichura dixanthia Hampson, $1898^{\mathrm{NEW}}$ & AM, Manaus* \\
\hline 257. & Trichura esmeralda (Walker, 1854) & AM, Anamã*; PA, [Belém] (Draudt 1915) \\
\hline 258. & Trichura latifascia (Walker, 1854) & PA, [Belém] (Walker 1854a), Santarém* \\
\hline 259. & Trichura mathina Druce, 1898 & $\begin{array}{l}\text { AM, [Eirunepé] \{Rio Juruá, São Felipe }\} \text { (Zerny 1931), São Paulo de Olivença*; } \\
\text { PA, [Belém] (Druce 1898), Santarém* }\end{array}$ \\
\hline 262. & Uranophora atalanta (Druce, 1899) & [AM], Amazonas [river] (Druce 1899) \\
\hline 263. & Uranophora felderi (Zerny, 1912) ${ }^{\mathrm{NEW}}$ & AM, Manicoré* \\
\hline 264. & Uranophora jynx (Hübner-Geyer, 1832) & AM, Amazonas [river] (Zerny 1912), Fonte Boa*, São Paulo de Olivença* \\
\hline 265. & Uranophora leucotela (Butler, 1876) & $\begin{array}{l}\text { AM, Manaus*; PA, Altamira \{Monte Santo }\} \text { (Teston \& Delfina 2010), Itaituba*, } \\
\text { Santarém \{Taperinha }\} \text { (Zerny 1931) }\end{array}$ \\
\hline 266. & Uranophora metamela (Dognin, 1911) & PA, [Belém] (Gaede 1926) \\
\hline 267. & $\begin{array}{l}\text { Uranophora quadrimaculata (Möschler, 1872) } \\
\text { NEw }\end{array}$ & AM, São Gabriel da Cachoeira* \\
\hline 268. & $\begin{array}{l}\text { Uranophora splendida (Herrich-Schäffer, } \\
[1854])^{\mathrm{AMZ}}\end{array}$ & AM, Tefé*; RO, Candeias do Jamari* \\
\hline 272. & Urolosia brodea (Schaus, 1896) & PA, Santarém \{Taperinha\} (Zerny 1931) \\
\hline 273. & Xanthopleura perspicua (Walker, 1856) & $\begin{array}{l}\text { AM, [Eirunepé] \{Rio Juruá, São Felipe\} (Zerny 1931), Manicoré*; PA, Altamira } \\
\text { \{Monte Santo } \text { (Delfina \& Teston 2013), [Belém] (Walker 1856), Belterra } \\
\text { \{National Forest of Tapajós\} (Freitas 2014), Novo Progresso \{Cachimbo\}*, } \\
\text { Parauapebas \{Serra Norte, Carajás }{ }^{*}, \text { Santarém*; RO, Cacaulândia* }\end{array}$ \\
\hline
\end{tabular}

$\overline{\mathrm{AMZ}}$ Species recorded for states within the Amazon biome by Ferro and Diniz (2010), but without precise location and biome information + species recorded in Ferro and Diniz (2007). So these species are new records for the municipalities. ${ }^{\mathrm{NEW}}$ New record for the Brazilian Amazon. 
Table 2. Geographic coordinates of municipalities and richness of the Ctenuchina species (Erebidae, Arctiinae, Arctini) in the Brazillian Amazon.

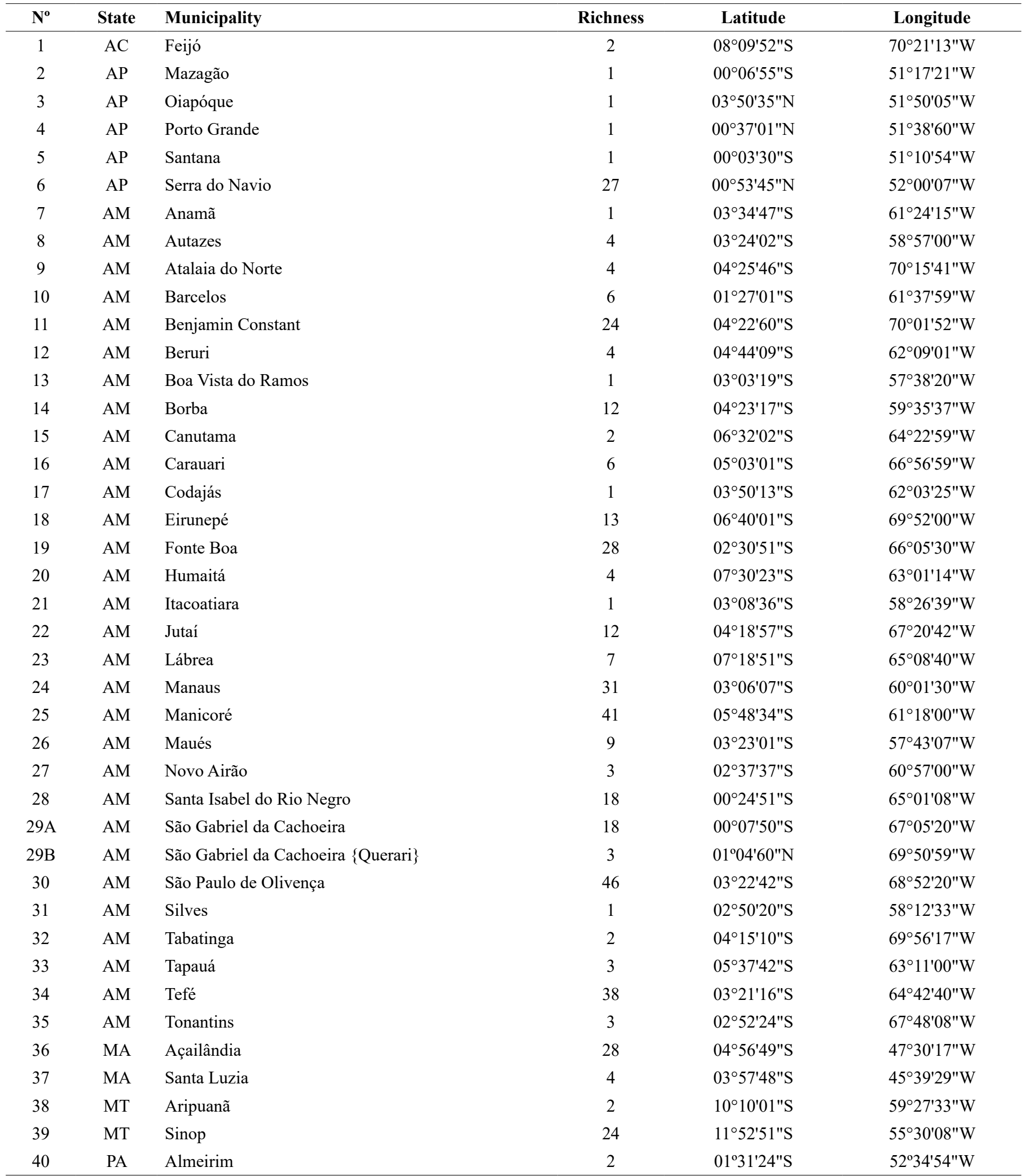


Teston, J.A. et al.

Continuation Table 2.

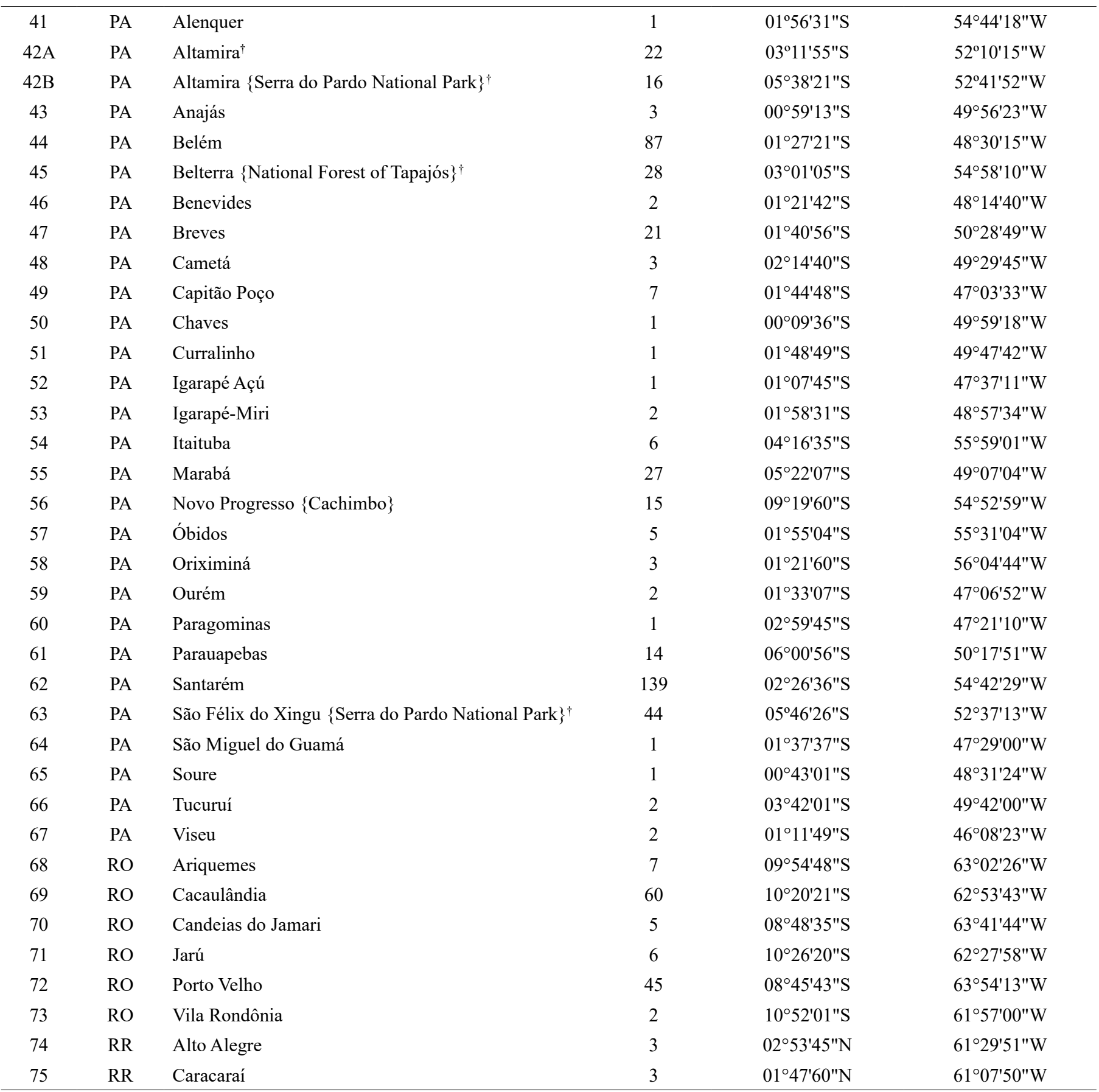

Geographic coordinates of municipality marked with ${ }^{\dagger}$ are the citations referred, other obtained by Google Earth or Geo Loc tool (see Materials and Methods). 


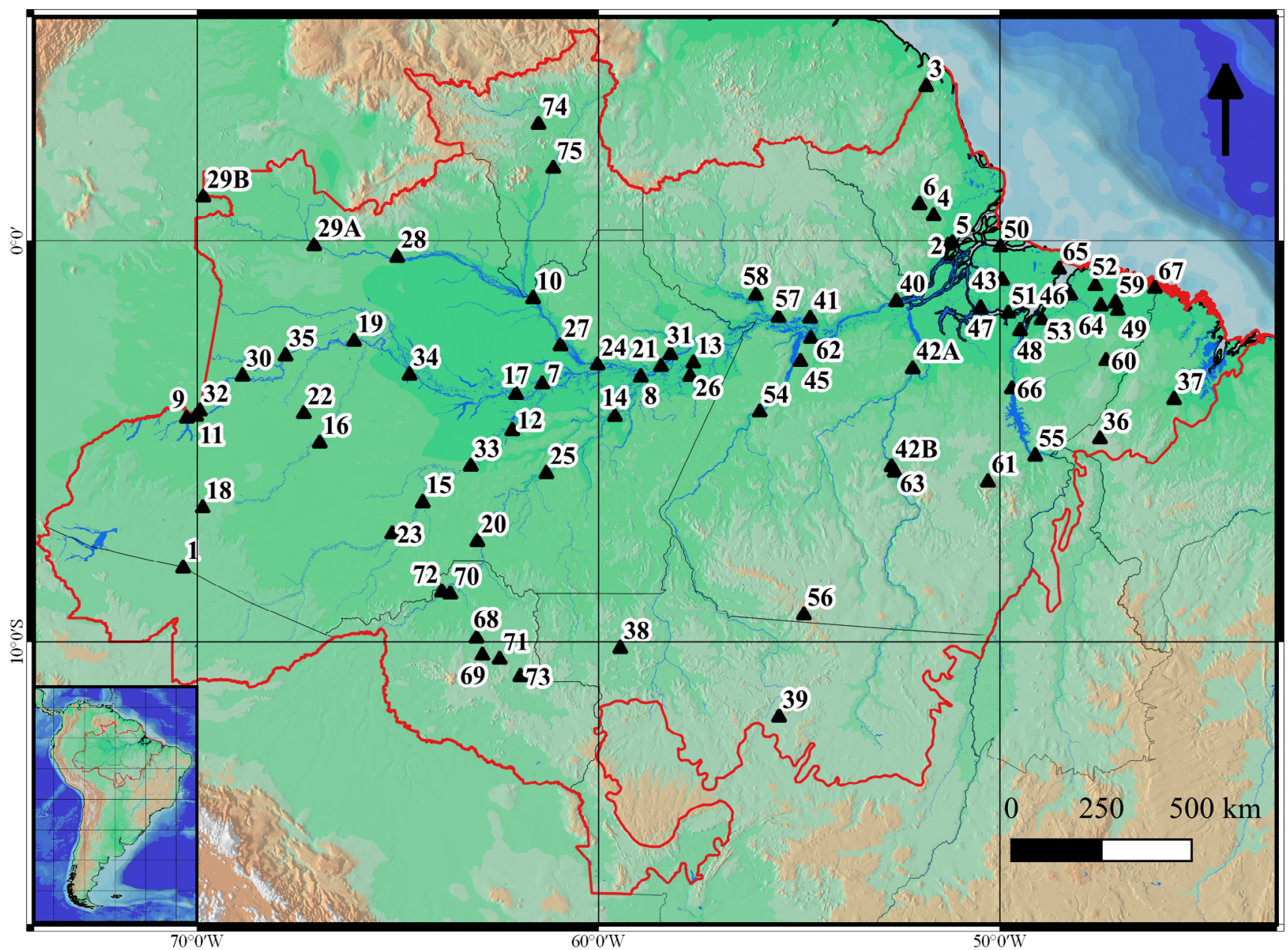

Figure 1. Geographic distribution of Ctenuchiina species records in Brazillian Amazon. The numbers refer to the municipalities of Table 2.

\section{Acknowledgements}

We are indebted to the museum curators for allowing VGF's access to the collections under their care and for logistical support. This publication is part of the RedeLep "Rede Nacional de Pesquisa e Conservação de Lepidópteros", SISBIOTA-Brasil, CNPq (563332/2010-7).

\section{Author contributions}

José A. Teston: Substantial contribution in the concept and design of the study.

Viviane G. Ferr: Substantial contribution in the concept and design of the study.

Débora S. de Abreu: Contribution to data collection.

\section{Conflicts of interest}

The authors declares that they have no conflict of interest related to the publication of this manuscript.

\section{References}

BRYK, F. 1953. Lepidoptera aus dem amazonasgebiete und aus Peru gesammelt von Dr. Douglas Melin und Dr. Abraham Roman. Ark. zool. 5(1): 1-268.

BUTLER, A.G. 1876. Notes on the Lepidoptera of the family Zygaenidae, whit descriptions of new genera and species. J. Linn. Soc. Lond., Zool. 12(60-62): 342-407.

BUTLER, A.G. 1877. Illustrations of typical specimens of Lepidoptera Heterocera in the British Museum part I. Taylor \& Francis, London.

BUTLER, A.G. 1878. On the Lepidoptera of the Amazons, collected by Dr James W. H. Trail during the years 1873 to 1875 . Trans. Entomol. Soc. Lond. 26(1): 39-84.

CONNER, W.E. \& JORDAN, A.T. 2009. From armaments to ornaments: the relationship between chemical defense and sex in tiger moths. In Tiger moths and wooly bears. Behavior, ecology, and evolution of the Arctiidae (W.E. Conner, ed.). Oxford University Press, New York, p.155-172.

DELFINA, M.C. \& TESTON, J.A. 2013. Arctiinae (Lepidoptera, Arctiidae) ocorrentes em uma área de pastagem na Amazônia Oriental em Altamira, Pará, Brasil. Acta Amaz. 43(1): 81-90.

DOGNIN, P. 1921. Hétérocères nouveaux de l'Amérique du Sud Fascicule XIX. Imprimerie Oberthür, Rennes.

DOGNIN, P. 1923. Hétérocères nouveaux de l'Amérique du Sud Fascicule XXIII. Imprimerie Oberthür, Rennes. 
DRAUDT, M. 1914-1919. Familie: Syntomidae. In Die Gross-Schmetterlinge der Erde. II. Abteilung: Die Gross-Schmetterlinge des Amerikanischen Faunengebietes. 6. Band. Die Amerikanischen Spinner und Schwärmer (A. Seitz, ed.). Alfred Kernen, Stuttgart, p.33-230.

DRUCE, H. 1884 (1881-1900). Insecta. Lepidoptera-Heterocera. Vol. I. In Biologia Centrali-Americana (F.D. Godman \& O. Salvin, eds.). H. R. Porter, London, p.1-490.

DRUCE, H. 1893. Descriptions of new species of Lepidoptera Heterocera from Central and South America. Proc. Zool. Soc. Lond. 1893: 280-311.

DRUCE, H. 1895. Descriptions of some new Species of Heterocera from the Eastern Islands and Tropical America. Ann. Mag. nat. Hist. 15(6): 41-50.

DRUCE, H. 1898. Descriptions of some new species of Syntomidae, chiefly in the Oxford Museum. Ann. Mag. nat. Hist. 7(1): 401-408.

DRUCE, H. 1899. Descriptions of some new species of Heterocera from Tropical America. Ann. Mag. nat. Hist. 7(3): 293-302.

FELDER, C. \& FELDER, R. 1864-1875. P1 75-120 (1874). In Reise der österreichischen fregatte novara um die Erde in den Jahren 1857, 1858, 1859 unter den Befehlen des Commodore B. Von Wüllestorf-Urbair. Zoologischer Theil, Zweiter Band, Zweite Abtheilung: Lepidoptera (C. Felder, R. Felder \& A.F. Rogenhofer, eds.). Staatsdruckerei, Vienna.

FERRO, V.G. \& DINIZ, I.R. 2007. Arctiidae (Insecta: Lepidoptera) da Estação Biológica de Boracéia (Salesópolis, São Paulo, Brasil). Biota Neotrop. 7(3): 331-338. http://dx.doi.org/10.1590/S1676-06032007000300033 (last access on $15 / 07 / 2018$ )

FERRO, V.G. \& DINIZ, I.R. 2010. Riqueza e composição das mariposas Arctiidae (Lepidoptera) no Cerrado. In Cerrado: conhecimento quantitativo como subsídio para as ações de conservação (I.R. Diniz, J. Marinho-Filho, R.B. Machado \& R. Cavalcanti, eds.). Editora Thesaurus, Brasília, p.255-313.

FERRO, V.G., MELO, A.S. \& DINIZ, I.R. 2010. Richness of tiger moths (Lepidoptera: Arctiidae) in the Brazilian Cerrado: how much do we know? Zoologia 27(5): 725-731.

FERRO, V.G., RESENDE, I.M.H. \& DUARTE, M. 2012. Arctiinae (Lepidoptera: Erebidae) do estado de Santa Catarina, Brasil. Biota Neotrop. 12(4): 166-180. http://dx.doi.org/10.1590/S1676-06032012000400018 (last access on $15 / 07 / 2018)$

FIELD, W.D. 1975. Ctenuchid moths of Ceramidia Butler, Ceramidiodes Hampson, and the Caca Species Group of Antichloris Hubner. Washington. Smithson. contrib. zool. 198(1): 1-44.

FREITAS, M.P. de. 2014. Estratificação vertical de Arctiini (Lepidoptera, Erebidae, Arctiinae) na Floresta Nacional do Tapajós, Amazônia Oriental, Pará, Brasil. Dissertação de Mestrado, Universidade Federal do Oeste do Pará, Santarém.

GAEDE, M. 1926. Amatiden des Berliner Zoologischen Museums (Lep.). Dtsch. entomol. z. 1926(2): 113-136.

HAGMANN, G. 1938. Syntomideos (Amatideos=Euchromideos) do Estado do Pará. In Livro Jubilar do Professor Lauro Travassos. Typographia do Instituto Oswaldo Cruz, Rio de Janeiro, p.185-194.

HAMPSON, G.F. 1898. Catalogue of the Lepidoptera Phalaenae in the British Museum. Catalogue of the Syntomidae in the collection of the British Museum. Taylor \& Francis, London.

HAMPSON, G.F. 1901. Catalogue of the Lepidoptera Phalaenae in the British Museum. Catalogue of the Arctiadae (Arctianae) and Agaristidae in the collection of the British Museum. Taylor \& Francis, London.

HAMPSON, G.F. 1914. Catalogue of the Lepidoptera Phalaenae in the British Museum. Supplement. Volume I. Catalogue of the Amatidae and Arctiadae (Nolinae and Lithosianae) in the collection of the British Museum. Taylor \& Francis, London.

HAWES, J., MOTTA, C. da S., OVERAL, W.L., BARLOW, J., GARDNER, T.A. \& PERES, C.A. 2009. Diversity and composition of Amazonian moths in primary, secondary and plantation forest. J. Trop. Ecol. 25(3): 281-300.

HEPPNER, J.B. 1991. Faunal regions and the diversity of Lepidoptera. Trop. Lep. 2(suppl. 1): 1-85.
HERNÁNDEZ-BAZ, F., MORON, M.A., CHAMÉ-VAZQUEZ, E. \& GONZÁLEZ, J.M. 2013. Ctenuchina and Euchromiina (Lepidoptera: Erebidae: Arctiinae) of Three Biological Research Stations of México's Ecosur. J. Lepid. Soc. 67(3): 145-155.

HERRICH-SCHÄFFER, G.A.W. [1855]. Sammlung neuer oder bekannter ausseuropaischer Schmetterlinge 1. G.J. Manz, Regensburg.

JACOBSON, N.L. \& WELLER, S.J. 2002. A cladistics study of the Arctiidae (Lepidoptera) by using characters of immatures and adults. Thomas Say Publications in Entomology, Lanham.

JANZEN, D. \& HALLWACHS, W. 2005. Philosophy, navigation and use of a dynamic database (ACG Caterpillars SRNP) for an inventory of the macrocaterpillar fauna, and its foodplants and parasitoids of the Area de Conservación Guanacaste (ACG), north-western Costa Rica. http://janzen.sas. upenn.edu (last access in 30/07/2018).

KITCHING, I.J. \& RAWLINS, J.E. 1999. The Noctuoidea. In Lepidoptera, moths and butterflies. Volume 1: evolution, systematics, and biogeography (N.P. Kristensen, ed. vol.). In Handbook of zoology. Volume IV Arthropoda: insect (M. Fischer, ed.). de Gruyter, Berlin, p.355-401.

MACHADO FILHO, J. \& RÊGO BARROS, A. 1970. Ctenuchidae Neotropicais V. Redescrição do gênero Argyrtidia Hampson, 1898 e descrição de nova espécie (Lepidoptera - Heterocera). Bol. Mus. Nac. 278(1): 1-17.

MORENO, C. \& FERRO, V.G. 2016. Arctiinae moths (Lepidoptera, Erebidae) of the Emas National Park, Goiás, Brazil. Biota Neotropica 16, p. e20150037. doi: http://dx.doi.org/10.1590/1676-0611-BN-2015-0037 (last access on 31/07/2018).

PINHEIRO, L.R. 2016. Re-evaluation of the identities of Eucereon punctatum (Guérin-Méneville, [1844]) and E. archias (Stoll, 1790), with a discussion on E. mitigatum Walker, 1857, rev. stat. (Lepidoptera, Erebidae, Arctiinae, Arctiini, Ctenuchina). Zoosystema 38(1): 127-140.

PINHEIRO, L.R. \& DUARTE, M. 2013. Taxonomic notes on Ctenuchina, Euchromiina, and Phaegopterina (Lepidoptera, Erebidae, Arctiinae, Arctiini). Fla. Entomol. 96(2): 351-359.

PINHEIRO L.R, \& GAAL-HASZLER, S. 2015. Illustrated catalogue of Neotropical Ctenuchina, Euchromiina and Pericopina types (Lepidoptera, Erebidae, Arctiinae, Arctiini) described by Hans Zerny, with discussion on their taxonomic status. Zootaxa. 3925(4): 505-535.

PINHEIRO, L.R. \& MAY, W. 2015. Revision of the Genus Pseudaethria Schaus (Lepidoptera, Erebidae). Neotrop. Entomol. 44(6): 588-595.

RÊGO BARROS, A. \& MACHADO FILHO, J. 1971. Estudo dos Ctenuchidae Neotropicais - I Sobre o gênero Hyaleucerea Butler, 1875 (Lepidoptera Heterecera). Arq. Mus. Nac. 54(1): 171-176.

ROTHSCHILD, L.W. 1910. Descriptions of new species of Arctiinae in the Tring Museum. Novit. zool. 17(1): 172-188.

ROTHSCHILD, L.W. 1911. New Syntomidae and Arctianae. Novit. zool. 18(1): 154-158.

ROTHSCHILD, L.W. 1912. New Syntomidae. Novit. zool. 19(1): 151-186.

SANTOS, N. 1939. Contribuição ao conhecimento dos Euchromiidae IV. Gênero Androcharta Felder, 1862. (Lepidoptera). Boletim Biológico 4(1): 87-97.

SCHERRER, S., FERRO, V.G., RAMOS, M.N. \& DINIZ, I.R. 2013. Species composition and temporal activity of Arctiinae (Lepidoptera: Erebidae) in two cerrado vegetation types. Zoologia 30(2): 200-210.

SEITZ, A. 1919-1925. 4. Familie: Arctiidae, Bärenspinner. In Die GrossSchmetterlinge der Erde. II. Abteilung: Die Gross-Schmetterlinge des Amerikanischen Faunengebietes. 6. Band. Die Amerikanischen Spinner und Schwärmer (A. Seitz, ed.). Alfred Kernen, Stuttgart, p.231-497.

SIMONS, R.B. 2009. Adaptative coloration and mimicry. In Tiger moths and wooly bears. Behavior, ecology, and evolution of the Arctiidae (W.E. Conner, ed.). Oxford University Press, New York, p.115-126.

STAUDINGER, O. 1894 Hochandine Lepidopteren. Dtsch. entomol. z. IRIS. 7(1): 43-100.

TESTON, J.A. \& CORREA, D. do C.V. 2015. The Arctiini (Lepidoptera, Erebidae, Arctiinae) fauna of the Serra do Pardo National Park, Pará, Brazil. Check List 11(2): 1-9. 
TESTON, J.A. \& DELFINA, M.C. 2010. Diversidade de Arctiinae (Lepidoptera, Arctiidae) em área alterada em Altamira, Amazônia Oriental, Pará, Brasil. Acta Amaz. 40(2): 387-396.

TESTON, J.A., NOVAES, J.B. \& ALMEIDA JUNIOR, J.O.B. 2012. Abundância, Composição e Diversidade de Arctinae (Lepidoptera, Arctiidae) em um fragmento de floresta na Amazônia Oriental em Altamira, PA, Brasil. Acta Amaz. 42(1): 105-114.

TESTON, J.A. \& FERRO, V.G. 2016a. Arctiini Leach, [1815] (Lepidoptera, Erebidae, Arctiinae) of the Brazilian Amazon. I - Subtribe Phaegopterina Kirby, 1892. Check List 12(2): 1-16

TESTON, J.A. \& FERRO, V.G. 2016b. Arctiini Leach, [1815] (Lepidoptera, Erebidae, Arctiinae) of the Brazilian Amazon. II - Subtribe Pericopina Walker, [1865]. Check List 12(6): 1-7.

TRAVASSOS, L. 1952. Contribuição ao conhecimento dos "Arctiidae". XXX, Espécies do gênero "Nelphe" H. Schaeffer, 1855. Rev. Bras. Biol. 12(4): 369-384. TRAVASSOS, L. 1959. Contribuição ao conhecimento dos "Arctiidae". XLII. Gênero Eucereon Huebner, 1819 (Lepidoptera, Heterocera). Mem. Inst. Oswaldo Cruz 57(2): 172-184

TRAVASSOS, L. 1964a. Contribuição ao conhecimento dos Arctiidae. XLIII. Descrição da genitália de cinco espécies dos gêneros Eucereon Hubner, 1819 e Eucereoides Travassos, 1961. Rev. Bras. Entomol. 11(1): 9-29.

TRAVASSOS, L. 1964b. Cobtribuição ao conhecimento dos Arctiidae (Lepidoptera-Heterocera) gênero Theages Walker 1855. An. Acad. Bras. Cienc. 36(2): 173-188.

TRAVASSOS FILHO, L. 1938. Contribuição ao conhecimento dos Euchromiidae. Gênero Corematura Butler, 1876. Mem. Inst. Oswaldo Cruz 33(2): 259-262.

TRAVASSOS FILHO, L. 1940. Contribuição à Zoogeografia dos Euchromidae Brasileiros. Arq. Zool. Est. São Paulo. 2(10): 281-297.

TRAVASSOS FILHO, L. 1952. Redescrição de Corematura Butler, 1876 e de suas duas espécies. (Lepidoptera Ctenuchidae). Arq. Zool. Est. São Paulo. 8(3): 89-108.

TRAVASSOS FILHO, L. 1953. Riccia, novo gênero para Corematura aliaria (Druce 1890) e descrição do Alótipo dessa espécie. (Lep. Ctenuchidae). Pap. Avulsos Dep. Zool./Secr. Agric. 11(17): 280-287.

TRAVASSOS FILHO, L. 1957. Contribuição ao conhecimento dos Ctenuchidae. (Lep.) VII- Gênero Dinia Walker, 1854. Arq. Zool. Est. São Paulo. 10(2) 186-207.

TRAVASSOS FILHO, L. \& URBAN, H. 1967. Bionomia de Dinia eaegrus (Cramer, 1779). (Lepidoptera - Ctenuchidae). Pap. Avulsos Zool. 20(18): $217-222$.
VALENTE, D.M.P., ZENKER, M.M. \& TESTON, J.A. 2018. Tiger-Moths in Savannas in Eastern Amazon: First Assessment of Diversity and Seasonal Aspects. Neotrop. Entomol. 47 (6): doi:10.1007/s13744-017-0579-y.

VINCENT, B. \& LAGUERRE, M. 2014. Catalogue of the Neotropical Arctiini Leach, [1815] (except Ctenuchina Kirby, 1837 and Euchromiina Butler, 1876) (Insecta, Lepidoptera, Erebidae, Arctiinae). Zoosystema 36(2): 137-533.

WALKER, F. 1854a. List of the specimens of lepidopterous insects in the collection of the British Museum. Part I. - Lepidoptera Heterocera. Edward Newman, London.

WALKER, F. 1854b. List of the specimens of lepidopterous insects in the collection of the British Museum. Part II. - Lepidoptera Heterocera. Edward Newman, London.

WALKER, F. 1856. List of the specimens of lepidopterous insects in the collection of the British Museum. Part VII. — Lepidoptera Heterocera. Edward Newman, London.

WALKER, F. [1865]. List of the specimens of lepidopterous insects in the collection of the British Museum. Part XXXI. - Supplement. Edward Newman, London.

WALKER, F. 1866. List of the specimens of lepidopterous insects in the collection of the British Museum. Part XXXV. - Supplement Part 5. Edward Newman, London.

WATSON, A., FLETCHER, D.S. \& NYE, I.W.B. 1995. Noctuoidea: Arctiidae, Cocytiidae, Ctenuchidae, dilobidae, Dioptidae, lymantriidae, Notodontidae, Strepsimanidae, Thaumetopoeidae \& Thyretidae. In the Generic names of moths of the world (I.W.B. Nye, ed.). The Natural Histori Museum, London, v.2, p.1-228.

WELLER, S.J., DACOSTA, M., SIMMONS, R.B., DITTMAR, K. \& WHITING, M. 2009. Evolution and taxonomic confusion in Arctiidae. In Tiger Moths and Wolly Bears, Behavior, Ecology and Evolution of the Arctiidae (W.E. Conner, ed.). Oxford University Press, New York, p.11-30.

WELLER, S.J., SIMMONS, R.B., BOADA, R. \& CONNER, W.E. 2000. Abdominal Modifications Occurring in Wasp Mimics of the CtenuchineEuchromiine Clade (Lepidoptera: Arctiidae). Ann. Entomol. Soc. Am. 93(4): 920-928.

ZAHIRI, R., HOLLOWAY, J.D., KITCHING, I.J., LAFONTAINE, J.D., MUTANEN, M. \& WAHLBERG, N. 2012. Molecular phylogenetics of Erebidae (Lepidoptera, Noctuiodea). Syst. Entomol. 37(1): 102-124.

ZERNY, H. 1912. Syntomidae. In Lepidopterorum Catalogus (H. Wagner, ed.). W. Junk, Berlin, p.1-179.

ZERNY, H. 1931. Ergebnisse einer zoologischen Sammerlreise nach Brasilien, insbesondere in das Amazonasgebiet, ausgeführt von Dr. H. Zerny. VII. Teil. Lepidoptera III: Die Syntomiden des Staates Pará. Ann. Nat. hist. Mus. Wien 45(1): 225-263. 